\title{
NEMS Resonators for Detection of Chemical Warfare Agents Based on Graphene Sheet
}

\author{
Nikola Anđelićc $\mathbb{D}^{1},{ }^{1}$ Zlatan Car $\mathbb{D}^{1},{ }^{1}$ and Marko Čanađija ${ }^{(\mathbb{D}}{ }^{2}$ \\ ${ }^{1}$ Department of Automation and Electronics, University of Rijeka, Faculty of Engineering, Vukovarska 58 51000, Rijeka, Croatia \\ ${ }^{2}$ Department of Engineering Mechanics, University of Rijeka, Faculty of Engineering, Vukovarska, 5851000 Rijeka, Croatia \\ Correspondence should be addressed to Marko Čanađija; marko.canadija@riteh.hr
}

Received 22 January 2019; Revised 2 May 2019; Accepted 22 May 2019; Published 17 June 2019

Academic Editor: Xiao-Qiao He

Copyright (C) 2019 Nikola Anđelić et al. This is an open access article distributed under the Creative Commons Attribution License, which permits unrestricted use, distribution, and reproduction in any medium, provided the original work is properly cited.

Graphene sheets are the basis of nanoelectromechanical (NEMS) mass resonators that are in recent years experimentally used for detection of specific gas molecules in air. The aim of this paper is to theoretically investigate the possibility of application of graphene sheets in detection of Chemical Warfare Agents (CWA's). By application of the nonlocal theory of elasticity and classical plate theory, equations that describe natural vibrations of the simply supported single-layer graphene sheet (SLGS) and double-layer graphene sheet (DLGS) were derived. In order to detect attached CWA molecule, the frequency shift method was applied. The results indicate that the proposed methodology could be successfully implemented in order to detect an attached CWA molecule. However, only specific mode shapes could be employed for such detection.

\section{Introduction}

Due to its extraordinary mechanical, electrical, and thermal properties graphene sheets have many potential applications such as reinforced materials, molecule sensors, and NEMS mass resonators [1]. For structural applications of graphene sheets, it is important to know their macroscopic properties. Therefore, experimental and theoretical analysis of graphene sheets are necessary in order to design optimal materials and devices. Nowadays a lot of resources and effort is focused on extensive research for possible application of graphene sheets in high-performance hybrid supercapacitors, optoelectronic devices, and various types of highperformance sensors. Although the graphene is material with extraordinary characteristics, studies $[2,3]$ are conducted to examine their potential impact on health and environment. Nanoelectromechanical systems or shortly NEMS are strong candidates for variety of applications in semiconductorbased technology and fundamental science $[4,5]$. NEMS resonators are used as the precision mass sensors which are used to weigh cells, biomolecules, and gas molecules [6-8]. A NEMS mass sensor relies on monitoring how the resonance frequency of the NEMS resonator changes when an additional mass is absorbed onto its surface. Today there is intense interest in implementation of the graphene sheet as the building block of the NEMS resonator based on their material properties such as low specific mass and high stiffness. The research [9] introduced a method for the large-scale production of the high-quality free-standing graphene NEMS in which the graphene top surface is continuously covered by the supporting polymer throughout the fabrication process. Although graphene sheet is highly sensitive material and used is for gas sensing applications in research [10] the effective, single-step method is shown to enhance its sensitivity.

Graphene sheets based on their structural features can be divided as Pristine Graphene (PG), Graphene Oxide (GO), and Reduced Graphene Oxide (RGO) [11]. Each of these groups of graphene sheets has distinct gas sensing capabilities. According to the research [12] GO proved to be promising candidate of gas detection under standard humidity conditions. Transducing mechanisms that can be used for detection of nanoparticles are optical [13, 14] (luminescence, absorption, polarization, and fluorescence), mass [15] (acoustic waves, absorption, and polarization), electrochemical [16] (conductometric, amperometric, potentiometric, and ion-sensitive), piezoelectric [17], and thermal. 
Today most commonly used transducing mechanisms are optical, electrochemical, and mass. The research [18] showed that two-dimensional structure of graphene makes electron transport through graphene highly sensitive to the adsorption of the gas molecules. When certain gas molecule attached itself on the graphene surface, this leads to change in the electrical conductivity. The conductivity change can be attributed to the change in the local carrier concentration induced by the surface adsorbates which act as electron donors or electron acceptors. NEMS with mass transducing mechanisms operate on the frequency shift principle. This means that resonant frequencies are determined for the system without attached nanoparticles and for the system with attached nanoparticles. Based on the difference between these two frequencies the type of chemical compound can be approximately determined. So far, the NEMS resonators based on graphene sheets were being used for investigating detection from theoretical and experimental point of view with simple chemical compounds such as $\mathrm{H}_{2}, \mathrm{H}_{2} 0, \mathrm{O}_{2}, \mathrm{CO}_{2}$, $\mathrm{CO}, \mathrm{NO}_{2}, \mathrm{Fe}, \mathrm{Co}, \mathrm{Ni}, \mathrm{Ru}, \mathrm{Rh}, \mathrm{Pd}$, Os, Ir, and Pt [11]. Although the graphene as a building block of gas sensor has high sensitivity to gas molecules the proof-of-principle comparison studies [19] showed that graphene sensing capabilities could be enhanced using a nanoporous substrate.

The tendency among researchers [20-22] is to apply the NEMS resonators in detection of more complex chemical compounds. In the focus of the research at hand is detection of Chemical Warfare Agents (CWA). According to Organization for the Prohibition of Chemical Weapons (OPCW) document [23] the CWAs are classified according to mechanism of toxicity in humans into blister agents, nerve agents, asphyxiants, choking agents, and incapacitating/behavior altering agents. Blister agents are sulfur mustard, nitrogen mustard and lewisite. According to NATO codes nerve agents can be categorized as G series agents, GB (sarin), GD (Soman), GA (Tabun), GF (cyclosarin), and V series agents, VE, VG, VM, and VX. The letter G represents the country of origin which is Germany and letter $\mathrm{V}$ represents Venomous. Asphyxiants agents are cyanogen chloride, hydrogen cyanide, and arsine. Chocking agents are chlorine, chloropicrin, and phosgene [24].

The goal of this research is to develop a theoretical framework that can be implemented into NEMS in order to detect presence of CWA. To the best of authors' knowledge, such a framework was not proposed in the literature so far. The concept relies on the small size effects observed in both one-dimensional [25] and two-dimensional structures [26] operating at nano- and microscale. In short, forces that are irrelevant at the macroscale become important at smaller scales. In that way, the stress state at some point does not depend only on the strain state at the same location, but on strains in whole neighborhood of the point in question. This approach in mechanics is known as the nonlocal theory. The theory is based on two main methodologies. Currently predominating one is based on gradients developed for either isothermal [26-28] or nonisothermal environments [29-31]. The newer approach is based on the integral approach $[32,33]$. Consequently, in this paper, the vibrations of simply supported graphene sheet (GS) with and without attached CWA molecule are analyzed using the gradient based nonlocal plate theory. In a nutshell, the nonlocal plate theory is an extension of the Kirchhoff-Love plate theory and is used to model mechanical response of single-layer graphene sheets (SLGS) and double-layer graphene sheets (DLGS). Today, the nonlocal theory is extensively applied in order to investigate mechanical and vibrational properties of SLGS and DLGS. In $[4,34]$ the authors used a continuum model to develop the equation which describes vibrations of multilayered graphene sheets (MLGSs) and in their investigation the formula is derived to predict the van der Waals (vdW) interaction. Large amplitude vibrations of multilayered GS were analyzed in [35] using Hamilton's principle in order to obtain the coupled nonlinear partial differential equations of motion which are based on the von Karman strain theory and Eringen theory of nonlocal continuum. The circular plate theory with vdW force was applied in [4] to investigate vibrations of the circular DLGS system. In [36] the higher order shear deformation theory is used to investigate buckling properties of single graphene sheet. Using this theory the authors developed closed-form solutions for critical buckling forces of the graphene sheets. In [37] shear deformation theory for buckling analysis of SLGS is developed using nonlocal differential constitutive relations of Eringen. Nonlocal elasticity theory was employed to investigate effects of small scale on buckling of the rectangular nanoplate. The equations of motion of the nonlocal theories are derived and solved via Navier's procedure for all edges simply supported boundary conditions. Obtained results are verified with the known results in the literature. The influences played by effects of nonlocal parameter, length, and thickness of the graphene sheets and shear deformation effect on the critical buckling load are studied. Verification studies show that the proposed theory is not only accurate and simple in solving the buckling nanoplates, but also comparable with the other higher order shear deformation theories which contain more number of unknowns.

To summarize, the main novelty of this paper is to investigate the possibility of utilizing SLGS and DLGS as sensing elements of NEMS resonators in detection of CWA molecules based on absolute and relative frequency shift method. In [1] the authors applied the nonlocal theory in order to obtain frequency equations of simply supported SLGS and DLGS systems used as the nanomechanical mass sensor. However, during the derivation of the frequency equation for simply supported DLGS system, the nonlocal parameter was neglected so the final equation for this system is reduced to the classical (local) form. The research in this paper starts from these existing results. After the derivation of the nonlocal equation which describes the vibrations of simply supported SLGS and DLGS, influence of the nonlocal parameter, geometry of SLGS and DLGS, and nanoparticle mass (CWA molecule) attached to the GS on vibrations of SLGS is analyzed and results are graphically represented. In this investigation the center of SLGS and DLGS upper plate is considered as a point of the attached CWA molecules. The influence of attached CWA molecule position on resonant frequencies of SLGS is also investigated. The vibrations of SLGS and DLGS with and without attached nanoparticle are 
determined and frequency shift as a function of the nonlocal parameter and nanoparticle mass is also analyzed.

\section{Nonlocal Theory or Elasticity}

In the general elasticity model, the stress components at any point depend only on the strain component at the same point. In the nonlocal elasticity theory, the stress field at a certain point is a function of the strain distribution over a certain representative volume of the material centered at that point. This theory is used to study several phenomena related to nanoscale materials. The equation of motion for nonlocal linear elastic solids can be written in the following form:

$$
\boldsymbol{t}_{i j}+\boldsymbol{f}_{i}=\rho \ddot{\boldsymbol{u}}_{i}
$$

where $\rho, \boldsymbol{f}_{i}, \boldsymbol{u}_{i}$, and $\boldsymbol{t}_{i j}$ are the mass density, applied body forces vector, displacement vector, and the nonlocal stress tensor, respectively. The nonlocal stress tensor can be written in the following form:

$$
\boldsymbol{t}_{i j}=\int_{V} \boldsymbol{\alpha}\left(\left|\boldsymbol{x}-\boldsymbol{x}^{\prime}\right|, e_{0} a\right) \boldsymbol{\sigma}_{i j}\left(\boldsymbol{x}^{\prime}\right) d V\left(\boldsymbol{x}^{\prime}\right)
$$

where $x$ is the reference point in the body and $e_{0} a$ is the nonlocal parameter or scale coefficient. According to [29] the material parameter $e_{0}$ is a constant appropriate to each material and it is estimated in a way that relations of the nonlocal elasticity model could provide approximation of atomic dispersion curves of plane waves with those of atomic lattice dynamics. The value of material parameter $e_{0}$ can be found by comparing results obtained using nonlocal theory with results obtained with molecular dynamics simulation and atomistic based techniques. The parameter $a$ can be the lattice parameter, granular size, distance between atoms or similar quantitiy. $\left|\boldsymbol{x}-\boldsymbol{x}^{\prime}\right|$ is the Euclidian distance and $V$ is the region occupied by the body. In previous equation the term $\boldsymbol{\alpha}\left(\left|\boldsymbol{x}-\boldsymbol{x}^{\prime}\right|, e_{0} a\right)$ represents the two-dimensional nonlocal modulus and is given as

$$
\boldsymbol{\alpha}\left(|x|, e_{0} a\right)=\frac{1}{2 \pi\left(e_{0} a\right)^{2}} K_{0}\left(\frac{\sqrt{\boldsymbol{x} \cdot \boldsymbol{x}}}{e_{0} a}\right),
$$

where $K_{0}$ is modified Bessel function. Integration of (3) over the entire domain will result in unity. If the value $e_{0} a$ in the kernel function $\boldsymbol{\alpha}$ is equal to 0 then the nonlocal elasticity is reduced to classical elasticity. In analysis of graphene sheets and carbon nanotubes according to [38] the most commonly used nonlocal kernel function is the one represented in (3) and will be also used in this paper.

A parameter $\sigma_{i j}$ in (2) represents the local stress tensor of the classical elasticity theory at any point $x^{\prime}$ in the body and follows from the usual constitutive relation:

$$
\sigma_{i j}\left(x^{\prime}\right)=\lambda \varepsilon_{k k}\left(x^{\prime}\right) \delta_{i j}+2 \mu \varepsilon_{i j}\left(x^{\prime}\right),
$$

where $\lambda$ and $\mu$ are Lame constants and $\varepsilon_{i j}$ is the strain tensor.

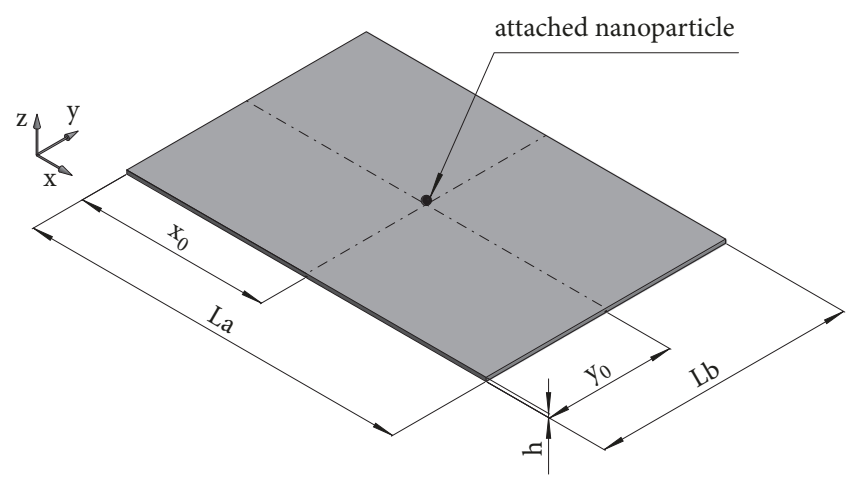

FIGURE 1: Schematic illustration of SLGS with attached mass at arbitrary position.

2.1. Single-Layer Graphene Sheets. As already mentioned, the dynamic behavior of a single-layer graphene sheet (SLGS) with the attached concentrated mass $m_{c}$ located at an arbitrary position $\left(x_{0}, y_{0}\right)$ is considered using nonlocal continuum mechanics. The SLGS is assumed to be simply supported at the boundary. The schematic illustration of the problem at hand is shown in Figure 1.

The origin of coordinate system is positioned in the lower left corner of the mid-plane of SLGS while $x$ and $y$ axes are oriented along the length $L_{a}$ and $L_{b}$, respectively. The out of plane axis $z$ is taken along the thickness $h$ of SLGS.

By applying the nonlocal elasticity theory [38] on SLGS the two-dimensional nonlocal constitutive equations are obtained and can be written in the following form:

$$
\begin{aligned}
& \sigma_{x x}-\left(e_{0} a\right)^{2}\left(\frac{\partial^{2} \sigma_{x x}}{\partial x^{2}}+\frac{\partial^{2} \sigma_{x x}}{\partial y^{2}}\right) \\
&= \frac{E}{1-v^{2}}\left(\varepsilon_{x x}+v \varepsilon_{y y}\right), \\
& \sigma_{y y}-\left(e_{0} a\right)^{2}\left(\frac{\partial^{2} \sigma_{y y}}{\partial x^{2}}+\frac{\partial^{2} \sigma_{y y}}{\partial y^{2}}\right) \\
&=\frac{E}{1-v^{2}}\left(\varepsilon_{y y}+v \varepsilon_{x x}\right), \\
& \tau_{x y}-\left(e_{0} a\right)^{2}\left(\frac{\partial^{2} \sigma_{x y}}{\partial x^{2}}+\frac{\partial^{2} \sigma_{x y}}{\partial y^{2}}\right)=G \gamma_{x y},
\end{aligned}
$$

where $E, G$, and $v$ are the elastic modulus, the shear modulus, and Poisson's ratio of the SLGS, respectively. The internal characteristic length $a$ corresponds to the distance between two atoms in a C-C bond and is equal to $0.142 \mathrm{~nm}$. Flexural moments of SLGS are obtained using the following expressions:

$$
M_{x x}=\int_{-h / 2}^{h / 2} z \sigma_{x x} d z,
$$




$$
\begin{aligned}
& M_{y y}=\int_{-h / 2}^{h / 2} z \sigma_{y y} d z \\
& M_{x y}=\int_{-h / 2}^{h / 2} z \tau_{x y} d z .
\end{aligned}
$$

If in-plane displacements of the middle surface are neglected in the $x$ and $y$ direction then the relationship between strain and displacement fields is expressed as

$$
\begin{gathered}
\varepsilon_{x x}=-z \frac{\partial^{2} w}{\partial x^{2}}, \\
\varepsilon_{y y}=-z \frac{\partial^{2} w}{\partial y^{2}}, \\
\gamma_{x y}=-2 z \frac{\partial^{2} w}{\partial x \partial y},
\end{gathered}
$$

where $w$ is displacement in direction of $z$ axis of GS. With substitution of (5a), (5b), and (5c) into (6) and using (7) the following expressions are obtained:

$$
\begin{gathered}
M_{x x}-\left(e_{0} a\right)^{2}\left(\frac{\partial^{2} M_{x x}}{\partial x^{2}}+\frac{\partial^{2} M_{x x}}{\partial y^{2}}\right) \\
=-D\left(\frac{\partial^{2} w}{\partial x^{2}}+v \frac{\partial^{2} w}{\partial y^{2}}\right) \\
M_{y y}-\left(e_{0} a\right)^{2}\left(\frac{\partial^{2} M_{y y}}{\partial x^{2}}+\frac{\partial^{2} M_{y y}}{\partial y^{2}}\right) \\
=-D\left(\frac{\partial^{2} w}{\partial y^{2}}+v \frac{\partial^{2} w}{\partial x^{2}}\right), \\
M_{x y}-\left(e_{0} a\right)^{2}\left(\frac{\partial^{2} M_{x y}}{\partial x^{2}}+\frac{\partial^{2} M_{x y}}{\partial y^{2}}\right) \\
=-D(1-v) G \frac{\partial^{2} w}{\partial x \partial y},
\end{gathered}
$$

where $D$ is the flexural rigidity of SLGS, expressed as

$$
D=\frac{E h^{3}}{12\left(1-v^{2}\right)} \text {. }
$$

The governing equation that describes the flexural vibration of SLGS carrying a nanoparticle at arbitrary position $\left(x_{0}, y_{0}\right)$ can be written in the following form:

$$
\begin{aligned}
& \frac{\partial^{2} M_{x x}}{\partial x^{2}}+2 \frac{\partial^{2} M_{x y}}{\partial x \partial y}+\frac{\partial^{2} M_{y y}}{\partial y^{2}} \\
& \quad=\left[\rho h+m_{c} \delta\left(x-x_{0}\right) \delta\left(y-y_{0}\right)\right] \frac{\partial^{2} w}{\partial t^{2}},
\end{aligned}
$$

where $\rho, m_{c}, t$, and $\delta$ are the mass density of SLGS, mass of nanoparticle, time, and the Dirac delta function, respectively.
With substitution of (8a), (8b), and (8c) into (10), the following governing equation is obtained:

$$
\begin{aligned}
& D\left(\frac{\partial^{4} w}{\partial x^{4}}+2 \frac{\partial^{4} w}{\partial x^{2} \partial y^{2}}+\frac{\partial^{4} w}{\partial y^{4}}\right) \\
& +\left[1-\left(e_{0} a\right)^{2}\left(\frac{\partial^{2} w}{\partial x^{2}}+\frac{\partial^{2} w}{\partial y^{2}}\right)\right] \\
& \cdot\left[\rho h+m_{c} \delta\left(x-x_{0}\right) \delta\left(y-y_{0}\right)\right] \frac{\partial^{2} w}{\partial t^{2}}=0 .
\end{aligned}
$$

The harmonic solution of previous equation can be expressed as

$$
w(x, y, t)=Y(x, y) e^{i \omega t}
$$

where $Y(x, y)$ represents a shape function of deflection and $\omega$ is the resonant frequency of the SLGS.

Substituting (12) into (11), the governing equation can be expressed as

$$
\begin{aligned}
& \left(\frac{\partial^{4} Y}{\partial x^{4}}+2 \frac{\partial^{4} Y}{\partial x^{2} \partial y^{2}}+\frac{\partial^{4} Y}{\partial y^{4}}\right) \\
& -\frac{\omega^{2}}{D}\left[1-\left(e_{0} a\right)^{2}\left(\frac{\partial^{2} Y}{\partial x^{2}}+\frac{\partial^{2} Y}{\partial y^{2}}\right)\right] \\
& \cdot\left[\rho h+m_{c} \delta\left(x-x_{0}\right) \delta\left(x-y_{0}\right)\right] \frac{\partial^{2} Y}{\partial t^{2}}=0 .
\end{aligned}
$$

Since the GS is simply supported, the following boundary conditions are applied:

$$
\begin{aligned}
w & =0 \\
\frac{\partial^{2} w}{\partial x^{2}} & =0 \\
\frac{\partial^{2} w}{\partial y^{2}} & =0
\end{aligned}
$$

on $x=0, L_{a}$, and $y=0, L_{b}$.

The shape function from (12) can be expressed as

$$
Y(x, y)=A_{m n} \sin \frac{m \pi x}{L_{a}} \sin \frac{n \pi y}{L_{b}},
$$

where parameter $A_{m n}$ represents the vibration amplitude and indexes $m$ and $n$ represent mode numbers in the periodic directions, respectively.

In order to obtain the equation for determining frequencies of SLGS, (15) is substituted in (13). After the substitution, the equation is multiplied by $x=\sin \left(m \pi x / L_{a}\right) \sin \left(n \pi y / L_{b}\right)$ and integrated over the whole region with respect to $x$ and $y$ 
in limits $x=0$ to $L_{a}$ and $y=0$ to $L_{b}$. With some simplifications the following frequency equation is obtained:

$$
\begin{aligned}
& \int_{0}^{L_{b}} \int_{0}^{L_{a}} A_{m n} D \pi^{4}\left(\frac{m^{2}}{L_{a}^{2}}+\frac{n^{2}}{L_{b}^{2}}\right)^{2} \sin ^{2} \frac{m \pi x}{L_{a}} \\
& \quad \cdot \sin ^{2} \frac{n \pi y}{L_{b}} d x d y \\
& \quad-\omega^{2} \int_{0}^{L_{b}} \int_{0}^{L_{a}} A_{m n}\left[1+\left(e_{0} a\right)^{2} \pi^{2}\left(\frac{m^{2}}{L_{a}^{2}}+\frac{n^{2}}{L_{b}^{2}}\right)\right]
\end{aligned}
$$

$$
\cdot\left[\rho h+m_{c} \delta\left(x-x_{0}\right) \delta\left(y-y_{0}\right)\right] \sin ^{2} \frac{m \pi x}{L_{a}} \sin ^{2} \frac{n \pi y}{L_{b}} d x d y
$$$$
=0 \text {. }
$$

The roots of the previous equation are the desired resonant frequencies and each resonant frequency corresponds to a given shape function. After further manipulation of the previous equation, the expression for determining resonant frequency is obtained in the following form:

$$
\omega_{m n}^{2}=\frac{D \pi^{4}\left(m^{2} / L_{a}^{2}+n^{2} / L_{b}^{2}\right)^{2}}{\left[1+\left(e_{0} a\right)^{2} \pi^{2}\left(m^{2} / L_{a}^{2}+n^{2} / L_{b}^{2}\right)\right]\left(\rho h+\left(4 m_{c} / L_{a} L_{b}\right) \sin ^{2} m \pi \xi \sin ^{2} n \pi \eta\right)},
$$

where $\xi=x_{0} / L_{a}$ and $\eta=y_{0} / L_{b}$ are the nondimensional parameters of nanoparticle position. In (17) in denominator $\sin ^{2} m \pi \xi \sin ^{2} n \pi \eta$ is multiplied by the nonlocal parameter $\left(e_{0} a\right)$ so the nonlocal parameter is influencing the mass localization on SLGS. The frequency is obtained from

$$
f_{m n}=\frac{\omega_{m n}}{2 \pi} \text {. }
$$

It is worth mentioning that when the nonlocal parameter $e_{0} a$ is equal to zero, the resonant frequency of SLGS with attached nanoparticles collapses to the classical plate theory.

2.2. Double-Layer Graphene Sheets. DLGS are composed of two SLGS and they interact with each other by vdW forces. In these simulations, the nanoparticle is attached on the upper layer while no nanoparticles are attached to the lower level of GS. The DLGS is assumed to be simply supported at the boundary. The DLGS system with attached nanoparticle at arbitrary position is shown in Figure 2.

The governing equation that describes vibration of this system can be written in the following form:

$$
\begin{aligned}
& D \nabla^{4} w_{1}+\left[1-\left(e_{0} a\right)^{2} \nabla^{2}\right] \\
& \cdot\left[\rho h+m_{c} \delta\left(x-x_{0}\right) \delta\left(y-y_{0}\right)\right] \frac{\partial^{2} w_{1}}{\partial t^{2}}=p_{12} \\
& D \nabla^{4} w_{2}+\rho h\left[1-\left(e_{0} a\right)^{2} \nabla^{2}\right] \frac{\partial^{2} w_{2}}{\partial t^{2}}=p_{21}
\end{aligned}
$$

where $w_{j}(x, y, t), j=1,2$ are the flexural deflections of upper $(j=1)$ and lower $(j=2)$ sheet and $p_{12}\left(p_{21}=-p_{12}\right)$ is the transverse pressure between layers of DLGS caused by vdW forces. As in the case of SLGS the variable $D$ is flexural rigidity given by (9) while $\nabla^{4}$ is the biharmonic operator and in the Cartesian coordinate system it can be written in the following form:

$$
\nabla^{4}(.)=\frac{\partial^{4}}{\partial x^{4}}+\frac{\partial^{4}}{\partial x^{2} \partial y^{2}}+\frac{\partial^{4}}{\partial y^{4}}
$$

The distributed transverse pressure acting on the upper and lower layer of DLGS system can be written as

$$
p_{12}=c\left(w_{2}-w_{1}\right)
$$

where $c$ is the vdW interaction coefficient between upper and lower layers $[4,34,35,40-42]$. It can be obtained from the Lennard-Jones potential as

$$
\begin{aligned}
c & =\left(\frac{4 \sqrt{3}}{9 a}\right)^{2} \frac{24 \varepsilon}{\sigma^{2}}\left(\frac{\sigma}{a}\right)^{8} \\
& \cdot\left[\frac{3003 \pi}{256} \sum_{k=0}^{5} \frac{(-1)^{k}}{2 k+1}\left(\begin{array}{l}
5 \\
k
\end{array}\right)\left(\frac{\sigma}{a}\right)^{6} \frac{1}{\left(\bar{z}_{1}-\bar{z}_{2}\right)^{12}}\right. \\
& \left.-\frac{35 \pi}{8} \sum_{k=0}^{2} \frac{(-1)^{k}}{2 k+1}\left(\begin{array}{l}
2 \\
k
\end{array}\right) \frac{1}{\left(\bar{z}_{1}-\bar{z}_{2}\right)^{6}}\right]
\end{aligned}
$$

where $\varepsilon=2.968 \mathrm{meV}$ and $\sigma=0.34 \mathrm{~nm}$ are parameters that are chosen to fit the physical properties of graphene sheets. The parameter $\bar{z}_{j}$ can be determined from the following equation:

$$
\bar{z}_{j}=\frac{z_{j}}{a}, \quad j=1,2
$$

where $z_{j}$ is the coordinate of $j$-th layer in the direction of the thickness with the origin in the mid-plane of the graphene sheets, and parameter $a=0.142 \mathrm{~nm}$ is the C-C bond length.

The second term in (19a) is given by

$$
\mu(x, y)=\rho h+m_{c} \delta\left(x-x_{0}\right) \delta\left(y-y_{0}\right)
$$

where $\mu(x, y)$ is the variable mass distribution function of the upper sheet. 


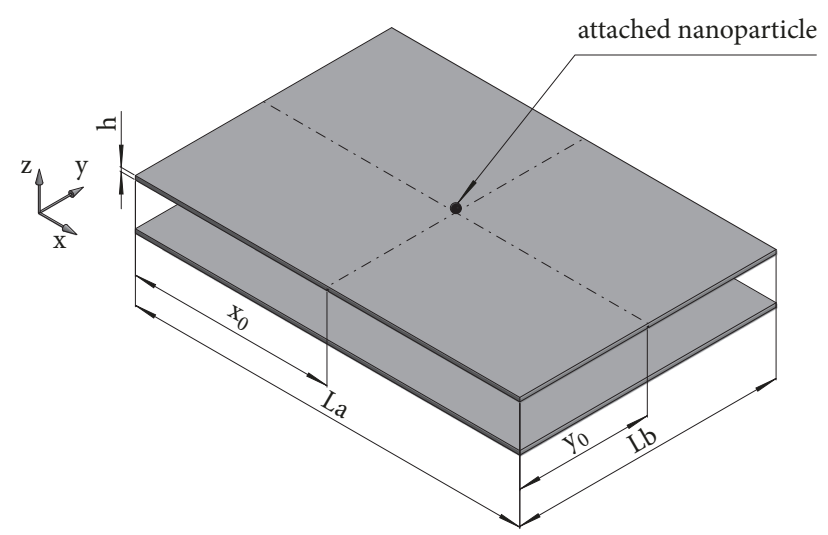

FIGURE 2: Schematic illustration of DLGS with attached mass at arbitrary position.
As in the case of SLGS, to obtain the resonant frequencies of DLGS system described by governing equations (19a) and (19b) the following function is introduced:

$$
w_{j}(x, y, t)=Y_{j}(x, y) e^{i \omega t}, \quad j=1,2
$$

where $Y_{j}(x, y), j=1,2$ represents a shape function of deflection in the upper and the lower sheet of DLGS system and $\omega$ is the resonant frequency.

$$
w_{j}(x, y, t)=Y_{j}(x, y) e^{i \omega t}, \quad j=1,2
$$

Substituting (26) into differential equations (19a) and (19b) of DLGS system the following equations are obtained in the matrix form:

$$
\left[\begin{array}{cc}
D \nabla^{4}-\mu \omega^{2}\left(e_{0} a\right)^{2} \nabla^{2}+c-\mu \omega^{2} & -c \\
-c & D \nabla^{4}-\rho h \omega^{2}\left(e_{0} a\right)^{2} \nabla^{2}+c-\rho h \omega^{2}
\end{array}\right]\left\{\begin{array}{l}
Y_{1} \\
Y_{2}
\end{array}\right\}=\left\{\begin{array}{l}
0 \\
0
\end{array}\right\}
$$

With further algebraic manipulation of the previous equation it is obtained that

$$
\begin{aligned}
\nabla^{8} Y_{j} & +\frac{(\mu+\rho h) \omega^{2}\left(e_{0} a\right)^{2}}{D^{2}} \nabla^{6} Y_{j} \\
& +\frac{\left(2 c-\rho h \omega^{2}-\mu \omega^{2}\right) D+\mu \rho h \omega^{4}\left(e_{0} a\right)^{4}}{D^{2}} \nabla^{4} Y_{j} \\
& +\frac{\left[c(\rho h+\mu)-2 \rho h \omega^{2}\right] \omega^{2}\left(e_{0} a\right)^{2}}{D^{2}} \nabla^{2} Y_{j} \\
& +\left[\frac{\rho h \mu \omega^{4}-c(\rho h+\mu) \omega^{2}}{D^{2}}\right] Y_{j}=0, \quad j=1,2 .
\end{aligned}
$$

Again, the DLGS system is simply supported which means that the following boundary conditions are applied:

$$
\begin{aligned}
w & =0 \\
\frac{\partial^{2} w}{\partial x^{2}} & =0 \\
\frac{\partial^{2} w}{\partial y^{2}} & =0
\end{aligned}
$$

$$
\text { on } x=0, L_{a} \text {, and } y=0, L_{b} \text {. }
$$

To fulfill previously defined boundary conditions, the following shape function of deflection is proposed:

$$
Y_{j}(x, y)=A_{m n} \sin \left(\frac{m \pi x}{L_{a}}\right) \sin \left(\frac{n \pi y}{L_{b}}\right), \quad j=1,2
$$

where $A_{m n}$ is the vibrational amplitude of oscillations, while indexes $m$ and $n$ indicate the mode numbers. With substitution of (30) into (28), the following equation is obtained:

$$
\begin{aligned}
\pi^{8} & \frac{\left(L_{b}^{2} m^{2}+L_{a}^{2} n^{2}\right)^{4}}{L_{a}^{8} L_{b}^{8}} Y_{j}-\pi^{6} \frac{\left(L_{b}^{2} m^{2}+L_{a}^{2} n^{2}\right)^{3}}{L_{a}^{6} L_{b}^{6}} \\
\cdot & \frac{(\mu+h \rho) \omega^{2}\left(e_{0} a\right)^{2}}{D} Y_{j}+\pi^{4} \frac{\left(L_{b}^{2} m^{2}+L_{a}^{2} n^{2}\right)^{2}}{L_{a}^{4} L_{b}^{4}} \\
\cdot & \frac{D\left(2 c-\omega^{2}(\mu+h \rho)\right)+\rho h \mu \omega^{4}\left(e_{0} a\right)^{2}}{D^{2}} Y_{j}+\pi^{2} \\
& \frac{\left(L_{b}^{2} m^{2}+L_{a}^{2} n^{2}\right)}{L_{a}^{2} L_{b}^{2}} \\
& \cdot \frac{\left(e_{0} a\right)^{2} \omega^{2}\left(2 h \mu \rho \omega^{2}-c(\mu+\rho h)\right)}{D^{2}} Y_{j} \\
+ & \frac{\omega^{2}\left(h \mu \rho \omega^{2}-c(\mu+\rho h)\right)}{D^{2}} Y_{j}=0 .
\end{aligned}
$$

Next step is to substitute (24) into the previous equation:

$$
\begin{gathered}
\pi^{8} \frac{\left(L_{b}^{2} m^{2}+L_{a}^{2} n^{2}\right)^{4}}{L_{a}^{8} L_{b}^{8}} Y_{j}-\pi^{6} \frac{\left(L_{b}^{2} m^{2}+L_{a}^{2} n^{2}\right)^{3}}{L_{a}^{6} L_{b}^{6}} \\
\cdot \frac{h \rho \omega^{2}\left(e_{0} a\right)^{2}}{D} Y_{j}+\pi^{4} \frac{\left(L_{b}^{2} m^{2}+L_{a}^{2} n^{2}\right)^{2}}{L_{a}^{4} L_{b}^{4}} \\
\cdot \frac{\left(2 c-\omega^{2} \rho h\right)}{D} Y_{j}+\pi^{2} \frac{\left(L_{b}^{2} m^{2}+L_{a}^{2} n^{2}\right)}{L_{a}^{2} L_{b}^{2}}
\end{gathered}
$$




$$
\begin{aligned}
& \cdot \frac{\left(e_{0} a\right)^{2} \omega^{2} \rho h}{D^{2}} Y_{j}+\frac{\omega^{2} c \rho h}{D^{2}} Y_{j} \\
& =\left\{\pi^{6} \frac{\left(L_{b}^{2} m^{2}+L_{a}^{2} n^{2}\right)^{3}}{L_{a}^{6} L_{b}^{6}} \frac{\omega^{2}\left(e_{0} a\right)^{2}}{D}+\pi^{4}\right. \\
& \cdot \frac{\left(L_{b}^{2} m^{2}+L_{a}^{2} n^{2}\right)^{2}}{L_{a}^{4} L_{b}^{4}} \frac{\omega^{2}-\rho h \omega^{4}\left(e_{0} a\right)^{4}}{D^{2}}-\pi^{2} \\
& \cdot \frac{\left(L_{b}^{2} m^{2}+L_{a}^{2} n^{2}\right)}{L_{a}^{2} L_{b}^{2}} \frac{\left(e_{0} a\right)^{2} \omega^{2}\left(2 h \rho \omega^{2}-c\right)}{D^{2}} \\
& \left.\cdot Y_{j}-\frac{\omega^{2}\left(h \rho \omega^{2}-c\right)}{D^{2}}\right\} m_{c} \delta\left(x-x_{0}\right) \delta\left(y-y_{0}\right) Y_{j} .
\end{aligned}
$$

After multiplication of the previous equation with the term $\sin \left(m \pi x / L_{a}\right) \sin \left(n \pi y / L_{b}\right)$ from both sides and with integration over the whole region with respect to $x$ and $y$ the following solution is obtained:

$$
r_{0} \omega^{4}+r_{1} \omega^{2}+r_{2}=0
$$

where the coefficients $r_{0}, r_{1}$, and $r_{2}$ are

$$
\begin{aligned}
r_{0} & =\frac{1}{8 D^{2} L_{a}^{4} L_{b}^{4}} h\left(L_{a}^{2} L_{b}^{2}+\left(n^{2} L_{a}^{2}+m^{2} L_{b}^{2}\right)\left(e_{0} a\right)^{2} \pi^{2}\right)^{2} \rho\left(2 \left(m_{c}\right.\right. \\
& \left.+h L_{a} L_{b} \rho\right)+m_{c}(-2 \cos (2 n \pi \eta)-2 \cos (2 m \pi \xi) \\
& +\cos (2 \pi(n \eta-m \xi))+\cos (2 \pi(n \eta+m \xi)))), \\
r_{1} & =-\frac{1}{2 D^{2} L_{a}^{6} L_{b}^{6}}\left(c_{i j} L_{a}^{4} L_{b}^{4}+D\left(n^{2} L_{a}^{2}+m^{2} L_{b}^{2}\right)^{2} \pi^{4}\right) \\
& +\left(m^{2} L_{b}^{2}\left(e_{0} a\right)^{2} \pi^{2}+L_{a}^{2}\left(L_{b}^{2}+n^{2}\left(e_{0} a\right)^{2} \pi^{2}\right)\right)\left(h L_{a} L_{b} \rho\right. \\
& \left.+2 m_{c} \sin (n \pi \eta)^{2} \sin (m \pi \xi)^{2}\right), \\
r_{2} & =\frac{\left(n^{2} L_{a}^{2}+m^{2} L_{b}^{2}\right)^{2} \pi^{4}\left(2 c_{i j} L_{a}^{4} L_{b}^{4}+D\left(n^{2} L_{a}^{2}+m^{2} L_{b}^{2}\right)^{2} \pi^{4}\right)}{4 D L_{a}^{7} L_{b}^{7}} .
\end{aligned}
$$

$\xi$ and $\eta$, as in the SLGS case, represent the nondimensional parameter of the attached nanoparticle position. $r_{0}$ and $r_{1}$ depend on the nonlocal parameter so the nonlocal parameter influences natural frequencies as well. The solution of (33) gives a term for determining natural frequencies of DLGS system with attached nanoparticle and can be expressed as

$$
\omega_{m n}^{2}=\frac{-r_{1}+\sqrt{r_{1}^{2}-4 r_{0} r_{2}}}{2 r_{0}},
$$

And, finally, the frequency is obtained from

$$
f_{m n}=\frac{\omega_{m n}}{2 \pi} .
$$

\section{Results and Discussion}

As already described, the considered nanomechanical resonator consists of simply supported SLGS or DLGS with the
TABLE 1: Geometrical and mechanical characteristics of SLGS.

\begin{tabular}{lc}
\hline Description & Value \\
\hline Length $[\mathrm{nm}]$ & 30 \\
\hline Width $[\mathrm{nm}]$ & 20 \\
\hline Thickness [nm] & 0.345 \\
\hline Internal characteristic length [nm] & 0.142 \\
\hline Modulus of elasticity [TPa] & 1.00 \\
\hline Density [kg/m ${ }^{3}$ ] & 2300 \\
\hline Poisson's number & 0.149 \\
\hline
\end{tabular}

attached nanoparticle. Unless otherwise stated, the dimensionless parameters $\xi$ and $\eta$ are equal to 0.5 . This means that the nanoparticle is at the center of the graphene sheet. Regarding mechanical properties of the graphene sheet in general Young's modulus is not yet precisely determined. To this day researchers used different approaches such as density function theory (DFT) [43], molecular dynamics $[44,45]$ or MD, continuum mechanics approach [46, 47], finite element modeling [48], and experimental investigation [49] in order to determine its value. All these analyses gave Young's modulus of graphene sheet around $1 \mathrm{TPa}$ which will be used as Young's modulus in our simulations. Geometrical and mechanical data of the simply supported graphene sheet is shown in Table 1.

The attached nanoparticles in these analyses are CWA molecules and the data for each CWA molecule is given in Table 2.

3.1. Influence of Different Parameters on Frequencies of SLGS. In order to investigate how an attached CWA molecule affects resonant frequencies of SLGS, influence of parameters such as the nonlocal parameter, geometry, and mass of attached nanoparticle needs to be investigated. The influence of the nonlocal parameter on natural frequencies will be investigated first. For the geometrical and mechanical parameters listed in Table 1 the influence of the nonlocal parameter on natural frequencies of simply supported SLGS for different mode shapes is shown in Figure 3.

As seen in Figure 3, when the value of the nonlocal parameter corresponds to 0 the frequency of all the mode shapes is at its highest. In other words, the highest natural frequencies are obtained in the case of local solutions. As the value of the nonlocal parameter increases the natural frequency starts to decrease. Looking at the range of the nonlocal parameter from 0 to $20 \mathrm{~nm}$, it is obvious that the value of the nonlocal parameter is strongly influencing the resonant frequency. In range from 20 to $50 \mathrm{~nm}$, the resonant frequency drop is much slower. In order to achieve natural frequencies of SLGS as in experimental investigations that are lower than $1 \mathrm{GHz}$ without considering any dissipative mechanisms the value of the nonlocal parameter must be greater than $20 \mathrm{~nm}$. Since the focus of this paper is not to determine the true value of the nonlocal parameter but to examine the possibility of using graphene in CWA molecule detection, the nonlocal parameter in further analyses will range from 1 to $4 \mathrm{~nm}$. 
TABLE 2: Technical data of CWA molecules [39].

\begin{tabular}{|c|c|c|c|}
\hline Name & Molecular Formula & Average Mass $[\mathrm{Da}=\mathrm{g} / \mathrm{mol}]$ & Mass of one molecule $[\mathrm{zg}]$ \\
\hline \multicolumn{4}{|c|}{ Type of Chemical Warfare Agent: Blister agent } \\
\hline Sulfur Mustard & $\mathrm{C}_{4} \mathrm{H}_{8} \mathrm{Cl}_{2} \mathrm{~S}$ & 159.077 & 264.16 \\
\hline Nitrogen Mustard HN-1 & $\mathrm{C}_{6} \mathrm{H}_{13} \mathrm{Cl}_{2} \mathrm{~N}$ & 170.08 & 282.431 \\
\hline Nitrogen Mustard HN-2 & $\mathrm{C}_{5} \mathrm{H}_{11} \mathrm{Cl}_{2} \mathrm{~N}$ & 156.053 & 259.138 \\
\hline Nitrogen Mustard HN-3 & $\mathrm{C}_{6} \mathrm{H}_{12} \mathrm{Cl}_{3} \mathrm{~N}$ & 204.53 & 337.977 \\
\hline Lewisite & $\mathrm{C}_{2} \mathrm{H}_{2} \mathrm{AsCl}_{3}$ & 207.318 & 344.268 \\
\hline \multicolumn{4}{|c|}{ Type of Chemical Warfare Agent: Nerve gases } \\
\hline \multicolumn{4}{|c|}{ G series } \\
\hline Sarin & $\mathrm{C}_{4} \mathrm{H}_{10} \mathrm{FO}_{2} \mathrm{P}$ & 140.093 & 232.635 \\
\hline Tabun & $\mathrm{C}_{5} \mathrm{H}_{11} \mathrm{~N}_{2} \mathrm{O}_{2} \mathrm{P}$ & 162.127 & 262.224 \\
\hline Soman & $\mathrm{C}_{7} \mathrm{H}_{16} \mathrm{FO}_{2} \mathrm{P}$ & 182.173 & 302.512 \\
\hline Cyclosarin & $\mathrm{C}_{7} \mathrm{H}_{14} \mathrm{FO}_{2} \mathrm{P}$ & 180.16 & 299.17 \\
\hline \multicolumn{4}{|c|}{ V series } \\
\hline VE & $\mathrm{C}_{10} \mathrm{H}_{24} \mathrm{NO}_{2} \mathrm{Ps}$ & 253.342 & 420.694 \\
\hline VG & $\mathrm{C}_{10} \mathrm{H}_{24} \mathrm{NO}_{3} \mathrm{Ps}$ & 269.341 & 447.261 \\
\hline VM & $\mathrm{C}_{10} \mathrm{H}_{22} \mathrm{NO}_{2} \mathrm{Ps}$ & 239.315 & 397.401 \\
\hline VX & $\mathrm{C}_{11} \mathrm{H}_{26} \mathrm{NO}_{2} \mathrm{Ps}$ & 267.368 & 443.985 \\
\hline \multicolumn{4}{|c|}{ Type of Chemical Warfare Agent: Asphyxiants } \\
\hline Cyanogen chloride & $\mathrm{CClN}$ & 61.470 & 102.076 \\
\hline Hydrogen Cyanide & $\mathrm{CHN}$ & 27.025 & 44.8771 \\
\hline Arsine & $\mathrm{H}_{3} \mathrm{As}$ & 77.9454 & 129.434 \\
\hline \multicolumn{4}{|c|}{ Type of Chemical Warfare Agent: Choking Agent } \\
\hline Chlorine & $\mathrm{Cl}_{2}$ & 70.906 & 117.745 \\
\hline Chloropicrin & $\mathrm{CCl}_{3} \mathrm{NO}_{2}$ & 164.375 & 272.957 \\
\hline Phosgene & $\mathrm{CCl}_{2} \mathrm{O}$ & 98.916 & 164.258 \\
\hline \multicolumn{4}{|c|}{ Type of Chemical Warfare Agent: Behavioral Agents/Incapacitating Agents } \\
\hline 3-Quinuclidinyl benzilate (BZ) & $\mathrm{C}_{21} \mathrm{H}_{23} \mathrm{NO}_{3}$ & 337.41 & 560.296 \\
\hline
\end{tabular}

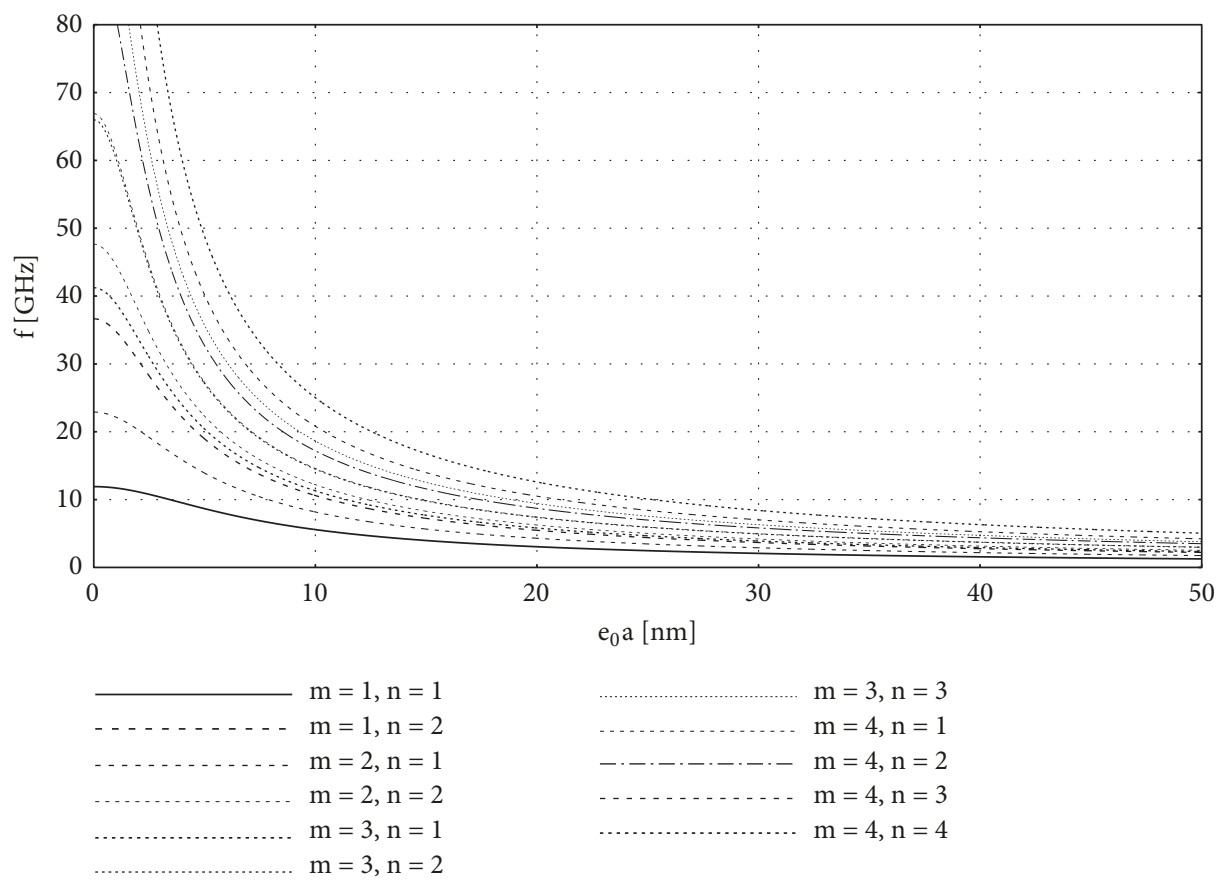

FIGURE 3: Variation of the natural frequency of SLGS versus nonlocal parameter for different mode shapes. 

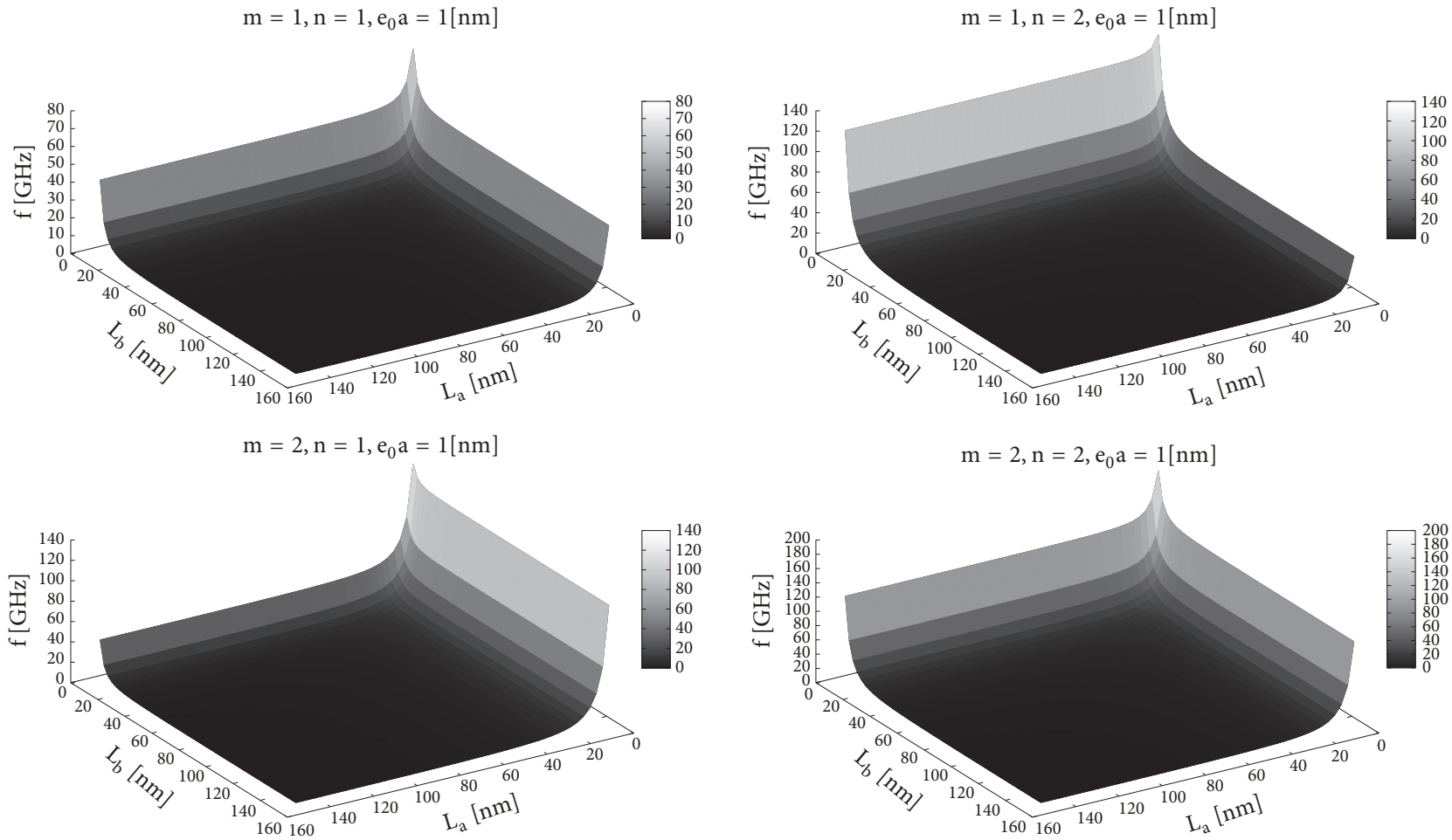

FIGURE 4: Variation of the natural frequency of the simply supported SLGS versus geometry $\left(e_{0} a=1 \mathrm{~nm}\right)$.

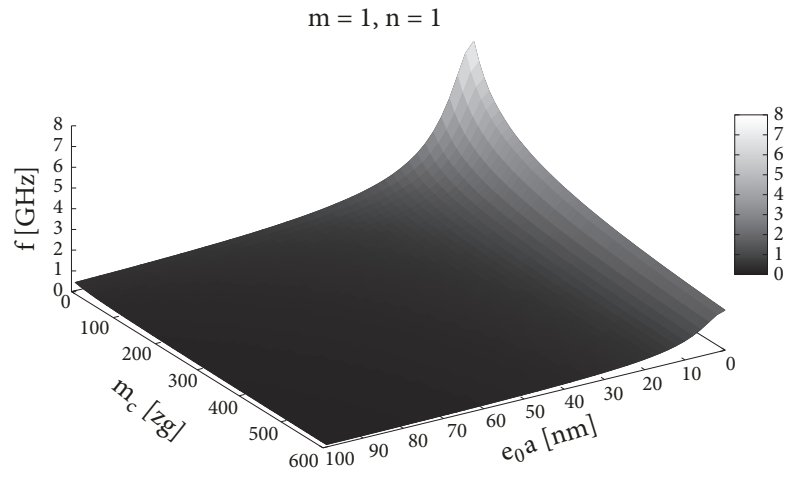

$\mathrm{m}=2, \mathrm{n}=1$

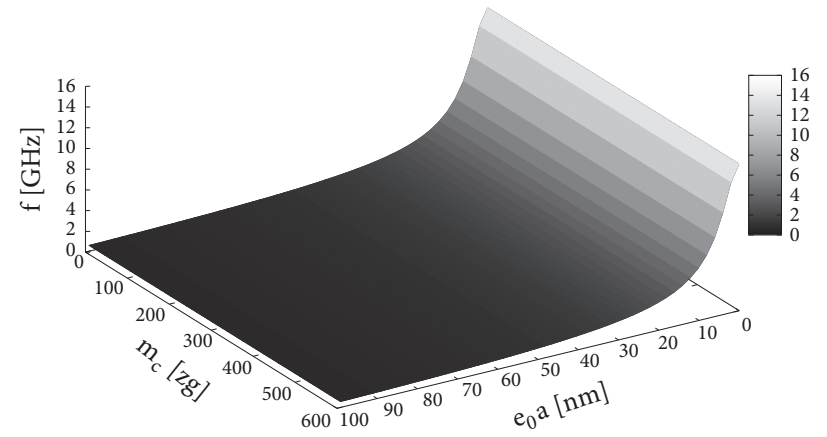

$\mathrm{m}=1, \mathrm{n}=2$

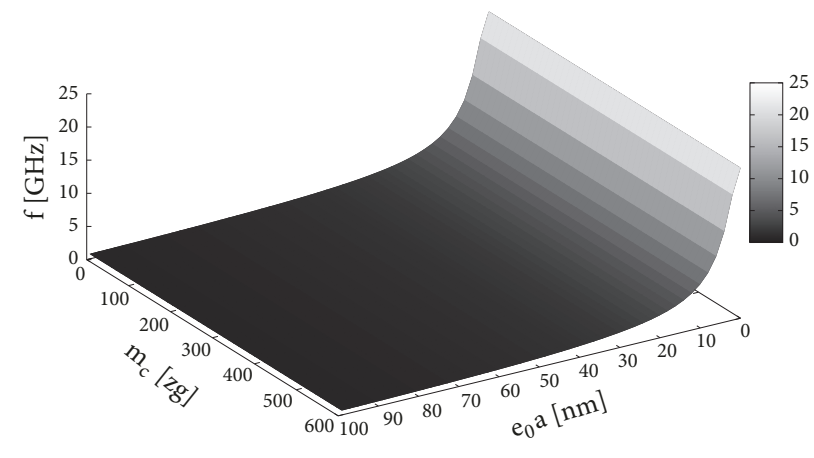

$\mathrm{m}=2, \mathrm{n}=2$

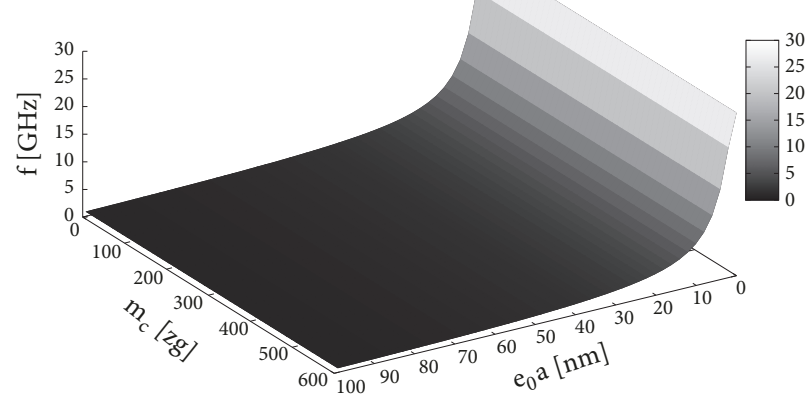

FIGURE 5: Influence of attached mass on resonant frequencies of SLGS $(\xi=0.5, \eta=0.5)$. 


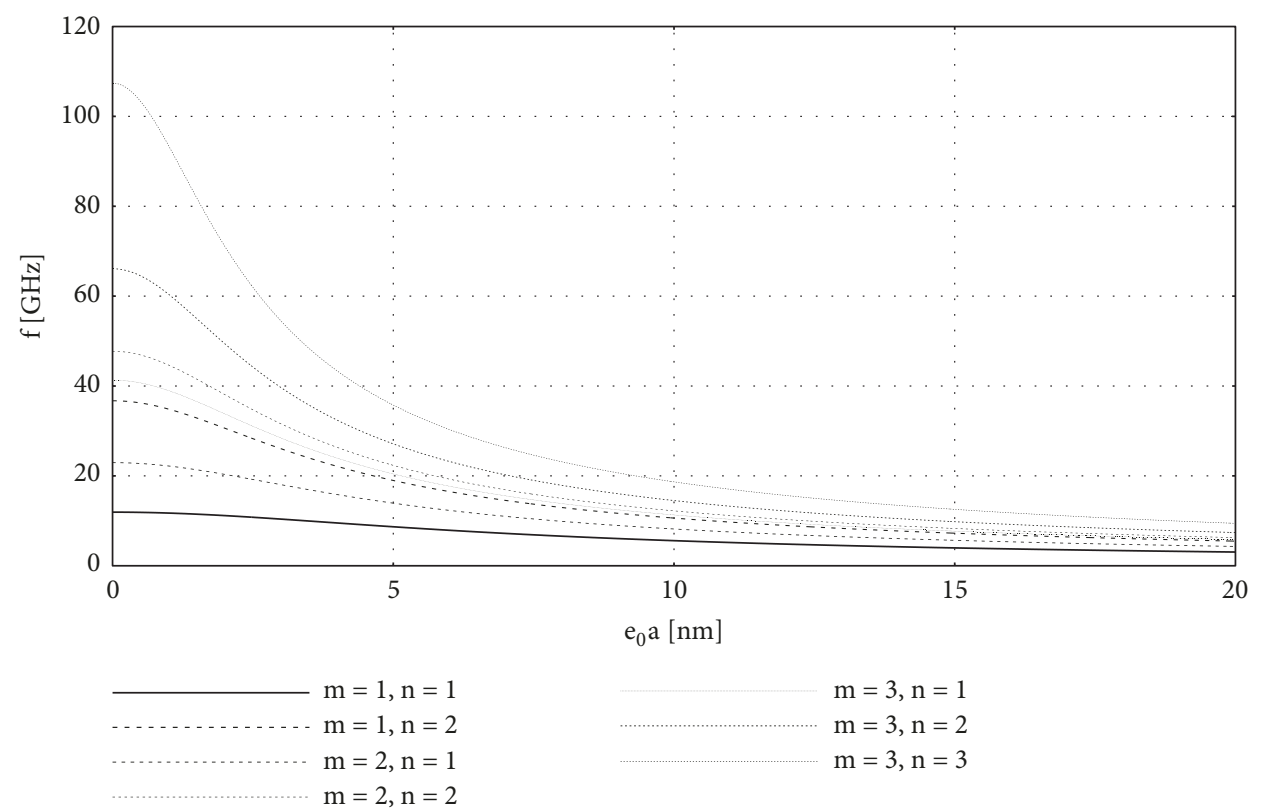

FIGURE 6: Natural frequencies of DLGS versus nonlocal parameter for different mode shapes.
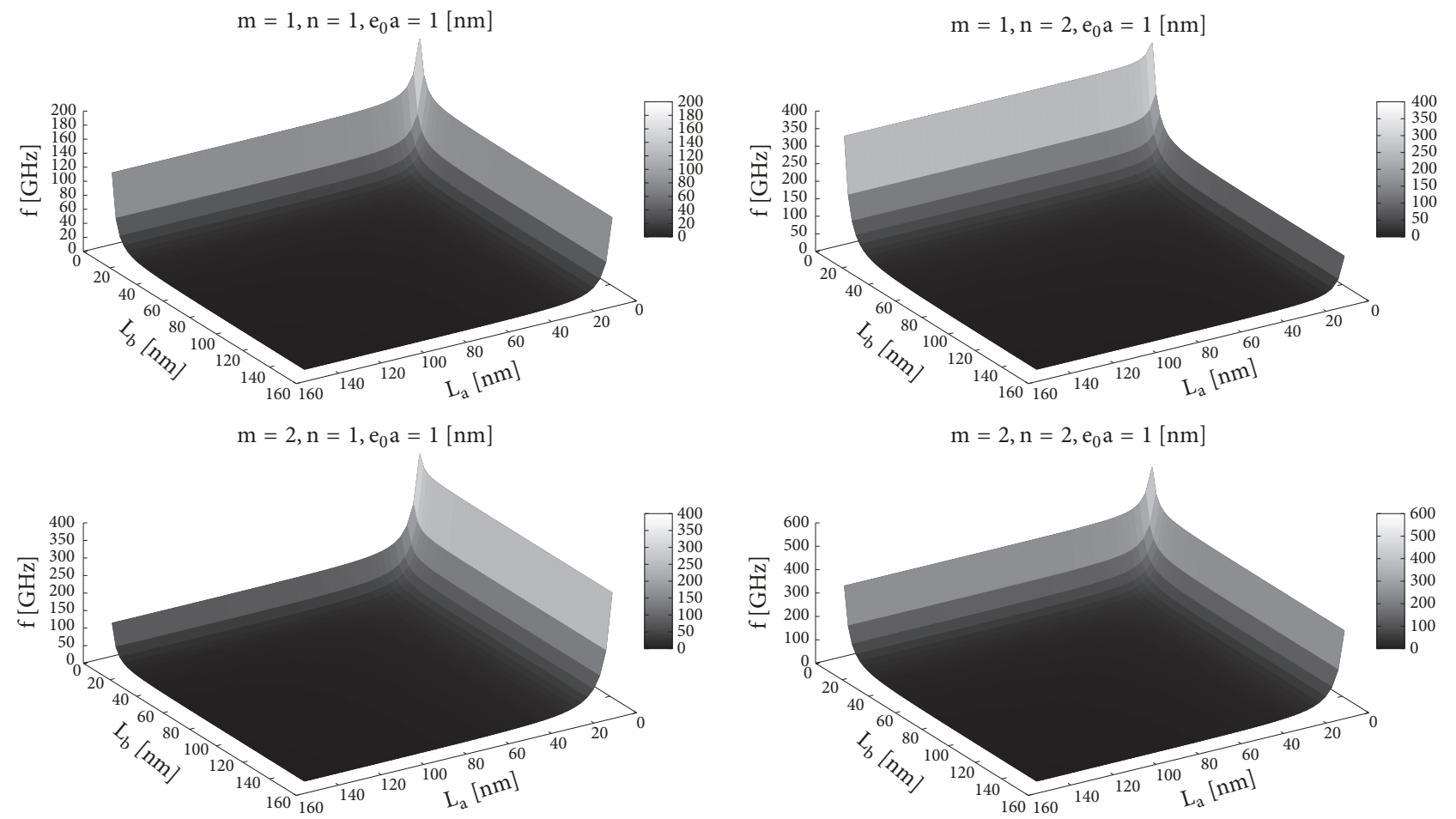

Figure 7: Natural frequency of DLGS versus its dimensions.

It should be emphasized that experimental investigations $[19,50,51]$ showed that resonant frequencies of graphene sheets are lower $(\mathrm{MHz}$ range) when compared to results obtained using the nonlocal theory of elasticity ( $\mathrm{GHz}$ range) $[1,4,31,34]$. This difference in frequency is due to the fact that the effect of all dissipative mechanisms on the experimental system is not known and because the exact value of the nonlocal parameter is still unknown, which represents the main disadvantage of the nonlocal theory for smaller values of the nonlocal parameter.

In order to investigate influence of geometry on the resonant frequency the mechanical characteristics from Table 1 

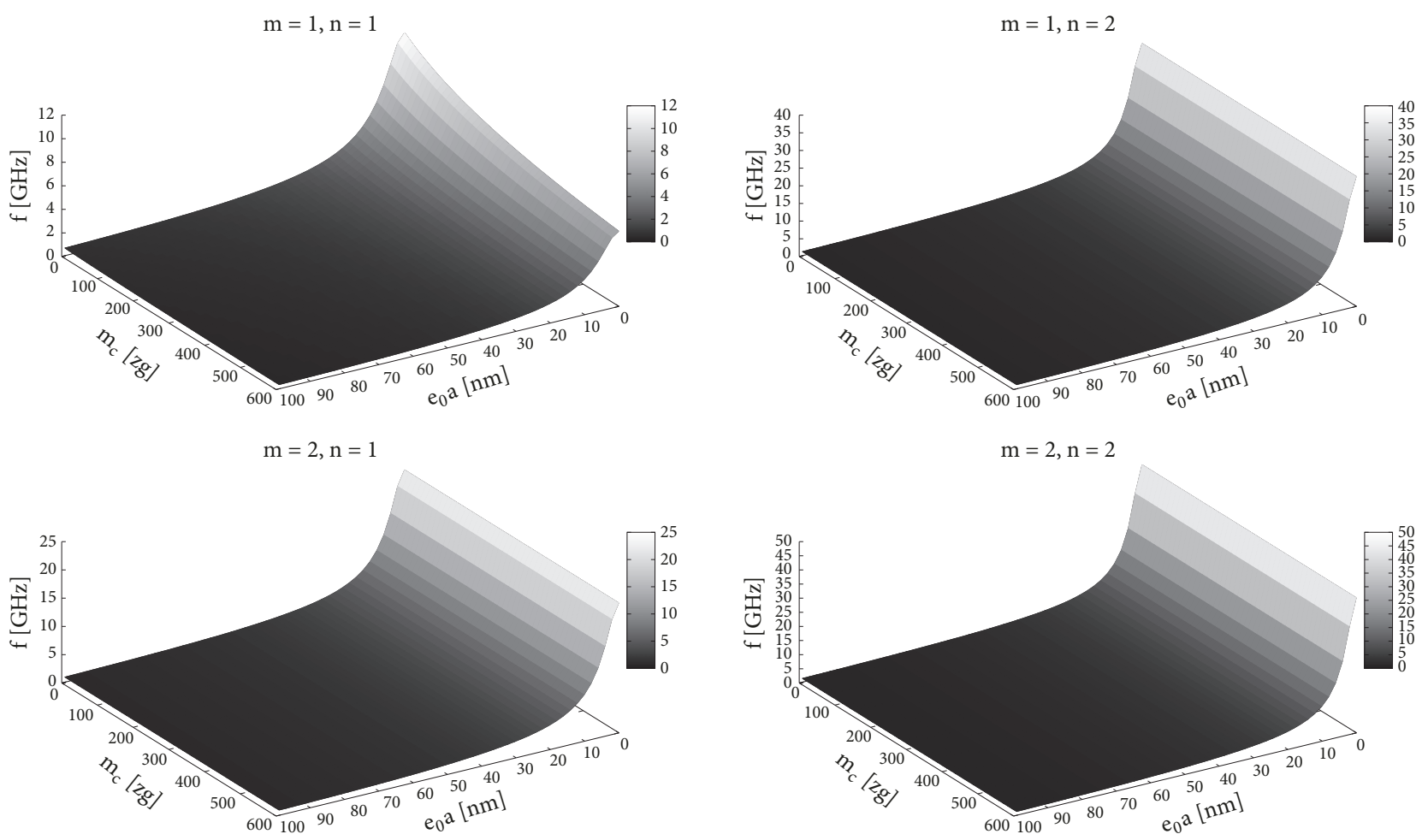

FIGURE 8: Resonant frequency of DLGS versus mass of the attached nanoparticle and nonlocal parameter $\left(e_{0} a=1[n m], \xi=0.5, \eta=0.5\right)$.

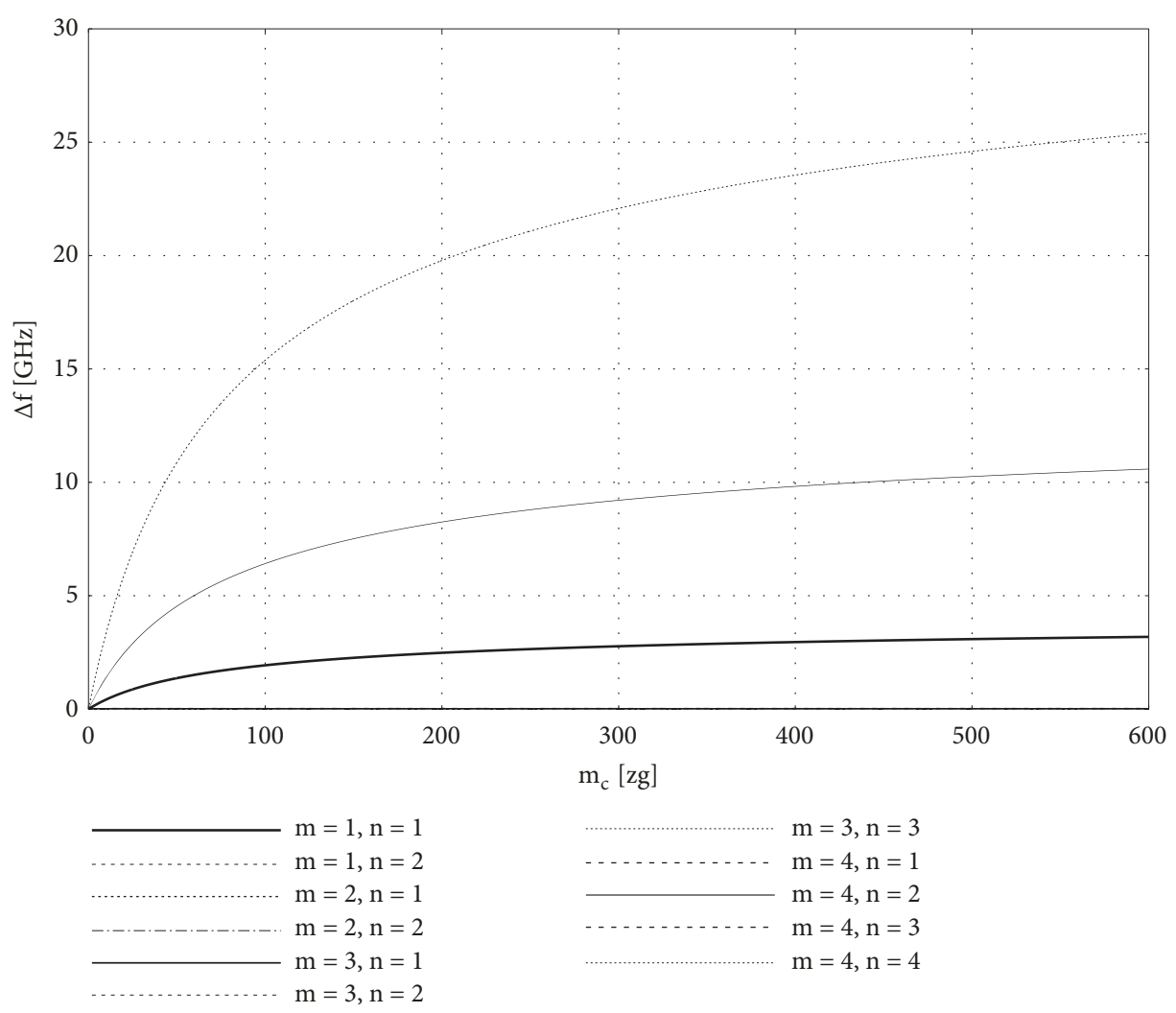

FIGURE 9: Variation of the frequency shift of simply supported SLGS versus attached mass for different mode shapes $\left(\xi=0.5, \eta=0.5, e_{0} a=\right.$ $1.0 \mathrm{~nm}$ ). 


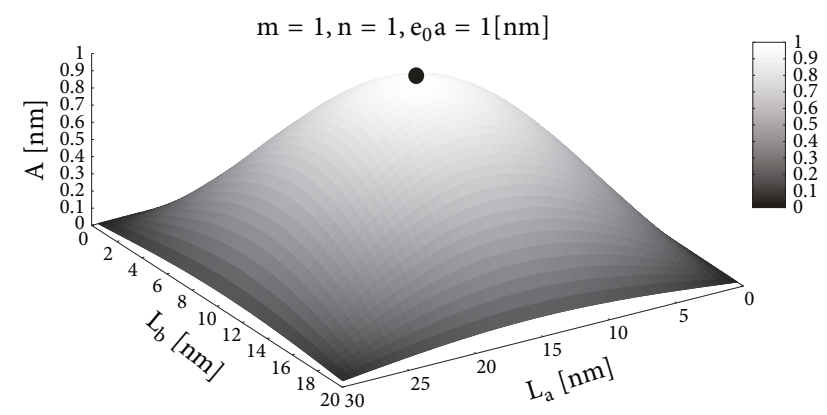

(a)

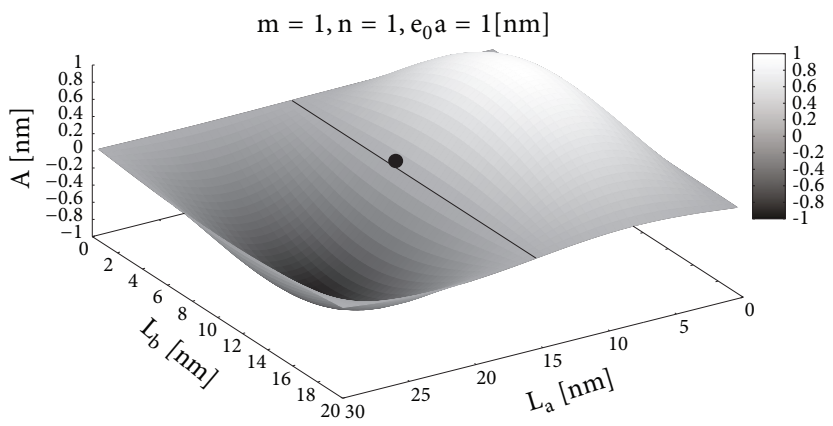

(b)

FIGURE 10: Two different mode shapes of simply supported SLGS: (a) mode shape without nodal lines $(m=1, n=1)$ and (b) mode shape with one nodal line $(m=2, n=1)$.

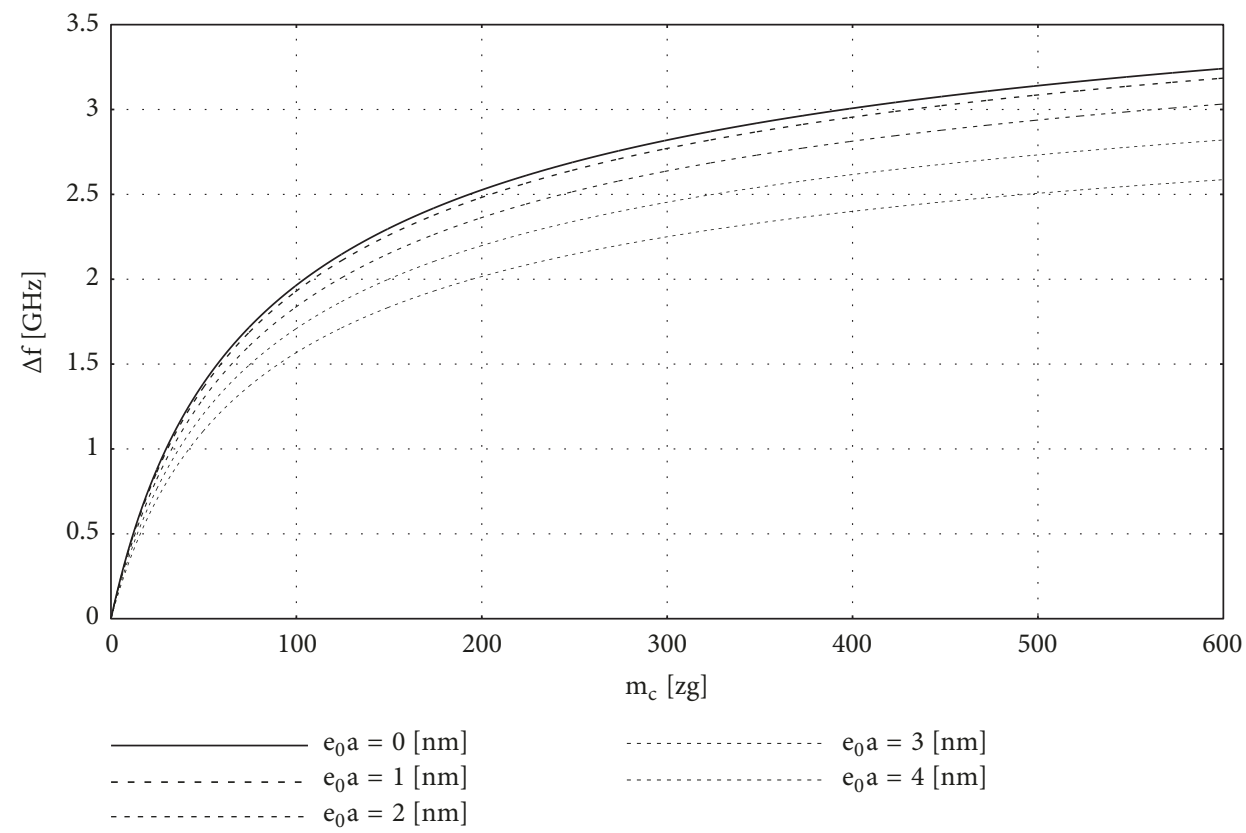

FIGURE 11: Variation of frequency shift of simply supported SLGS as a function of attached mass for different nonlocal parameters $(m=1, n=$ $1, \xi=0.5, \eta=0.5)$.

are taken into account and for this analysis the value of nonlocal parameter is $1 \mathrm{~nm}$ and values of nondimensional parameters of shape function of $m$ and $n$ are either 1 or 2 .

As seen from Figure 4 when the lengths $L_{a}$ and $L_{b}$ are in range from 5 to $20 \mathrm{~nm}$ then the natural frequencies are in range of 200 to $20 \mathrm{GHz}$. As the length of graphene sheet gets larger the frequency starts to drop down and is in range from 0 to $2 \mathrm{GHz}$.

To investigate the influence of the attached nanoparticle mass on resonant frequencies of the simply supported SLGS, the particle is placed at the center of SLGS, $\xi=0.5, \eta=0.5$. Since the mass of CWA molecules is in range from 0 to $600 \mathrm{zg}$ (Table 2), the mass of attached nanoparticle is varied in that range, respectively. For this simulation the mechanical and geometrical data are again taken from Table 1 and results of simulation for different mode shapes are shown in Figure 5.
From Figure 5 it is obvious that the nonlocal parameter has greater influence on the resonant frequency than the attached nanoparticle. Analyzed mode shapes points out that if the mass of attached nanoparticle and the nonlocal parameter is low the resonant frequency is around 8 $30 \mathrm{GHz}$ depending on the mode shape. As the mass of the attached nanoparticle and the nonlocal parameter gets larger the resonant frequency drops down to a $\mathrm{MHz}$ range which is closer to experimentally obtained results $[19,50$, 51].

3.2. Influence of Different Parameters on Frequencies of DLGS. For analysis of influence of different parameters on resonant frequencies of the simply supported DLGS the mechanical and geometrical parameters are used from Table 1. Firstly, the influence of the nonlocal parameter on natural frequencies is 


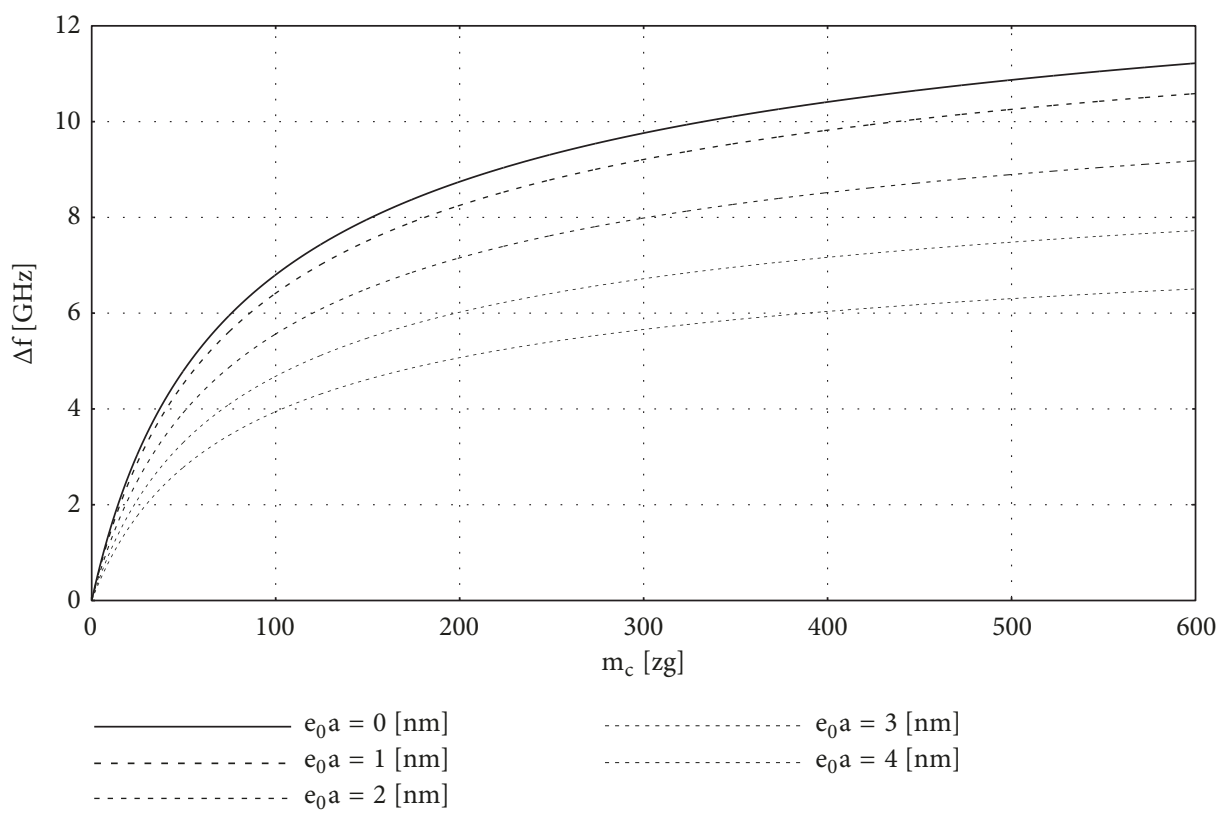

FIGURE 12: Variation of frequency shift of simply supported SLGS as a function of attached mass for different nonlocal parameters $(m=3, n=$ $1, \xi=0.5, \eta=0.5)$.

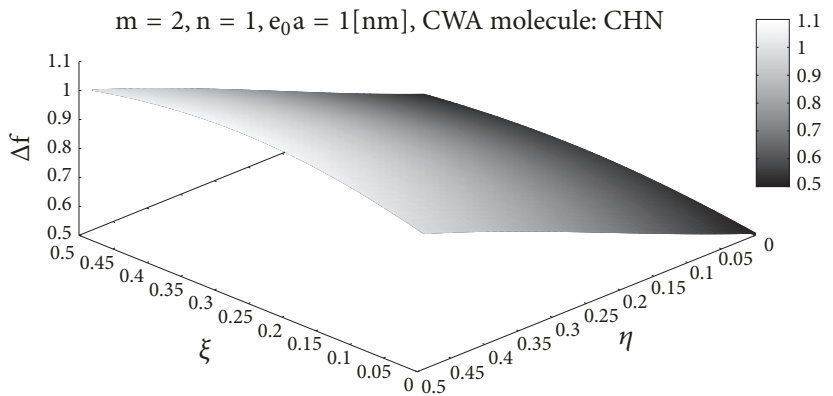

FIGURE 13: Influence of the nondimensional location parameters $\xi, \eta$ on the frequency shift for the case of attached hydrogen cyanide on the simply supported SLGS.

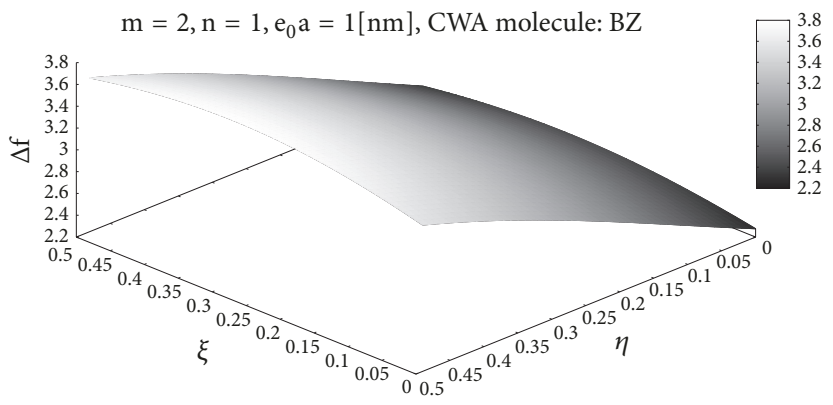

FIGURE 14: Influence of the nondimensional location parameters $\xi, \eta$ on the frequency shift for the case of attached 3-quinuclidinyl benzilate (BZ) on the simply supported SLGS.

investigated. In this analysis the value of nonlocal parameter ranges from 0 to $50 \mathrm{~nm}$, the mass of attached nanoparticle is equal to 0 and the nondimensional shape function parameters $m$ and $n$ are set in range from 1 to 4 . The result of calculation is shown in Figure 6.
As expected, the natural frequency of DLGS system is highest when the value of nonlocal parameter is equal to $0 \mathrm{~nm}$, i.e., for the local formulation. From Figure 6 it is obvious that resonant frequency starts to drop as the nonlocal parameter increases. 


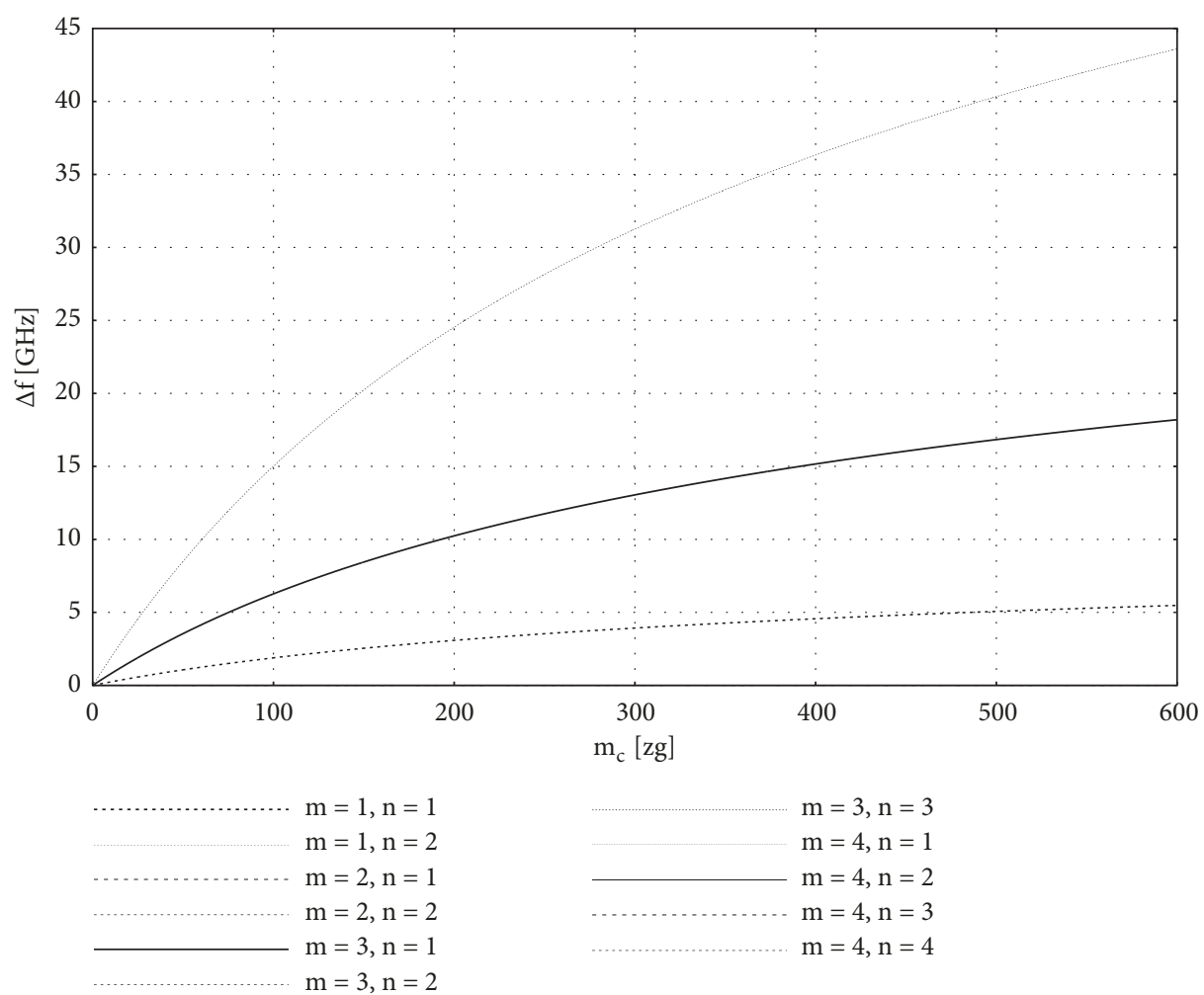

FIGURE 15: Variation of the frequency shift as a function of the attached nanoparticle mass on DLGS system for different mode shapes $\left(e_{0} a=\right.$ $1 \mathrm{~nm}, \xi=0.5, \eta=0.5)$.

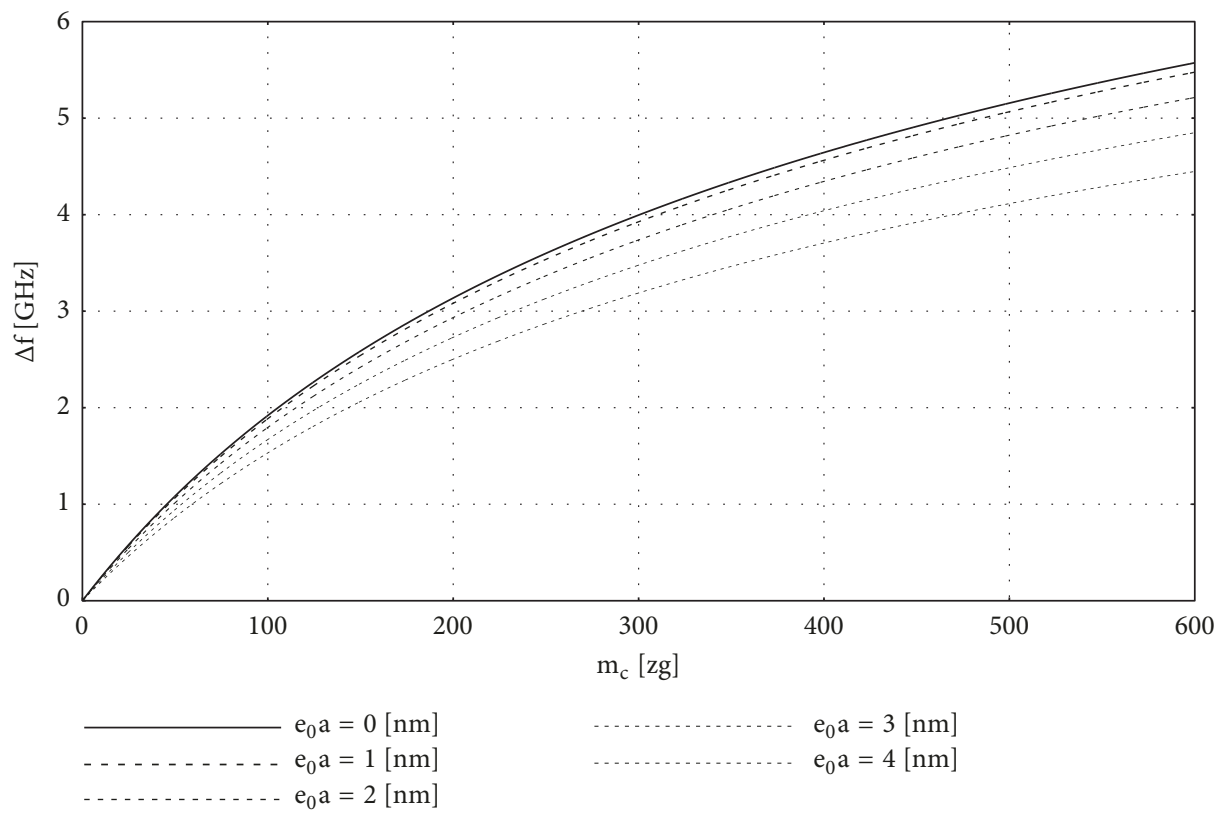

FIGURE 16: Variation of the frequency shift of the simply supported DLGS as a function of attached mass for different nonlocal parameters $(m=1, n=1, \xi=0.5, \eta=0.5)$.

The next step is to investigate the influence of geometry on natural frequencies of DLGS. For this analysis the nonlocal parameter is set to $1 \mathrm{~nm}$ and mechanical parameters are taken from Table 1. Results of the performed analysis are shown in Figure 7.
In Figure 7 the influence of geometry of simply supported DLGS system on natural frequency is shown. For each mode shape when the values $L_{a}$ and $L_{b}$ are small (in range from 5 to $40 \mathrm{~nm}$ ) the natural frequency of DLGS system is in range from 20 to $600 \mathrm{GHz}$ depending on the mode shape. When $L_{a}$ 


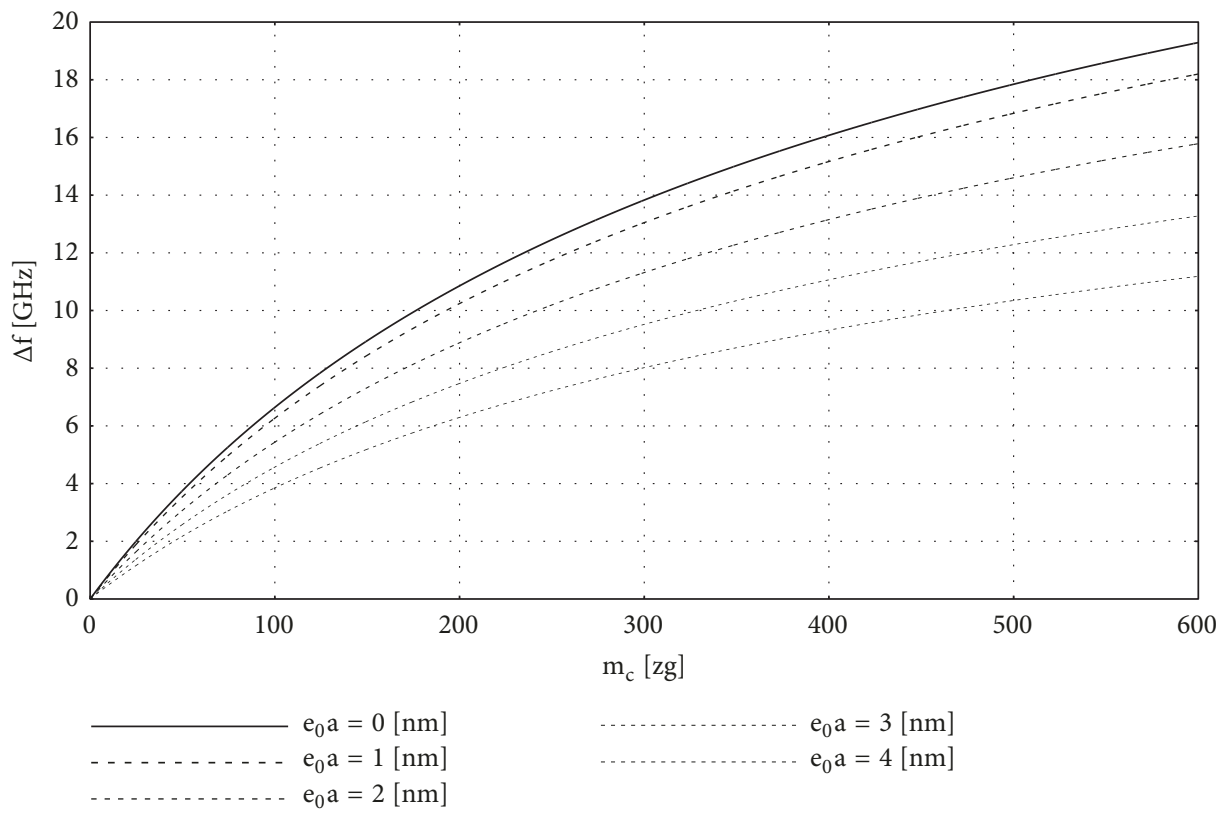

FIGURE 17: Variation of the frequency shift of the simply supported DLGS as a function of attached mass for different nonlocal parameters $(m=3, n=1, \xi=0.5, \eta=0.5)$.

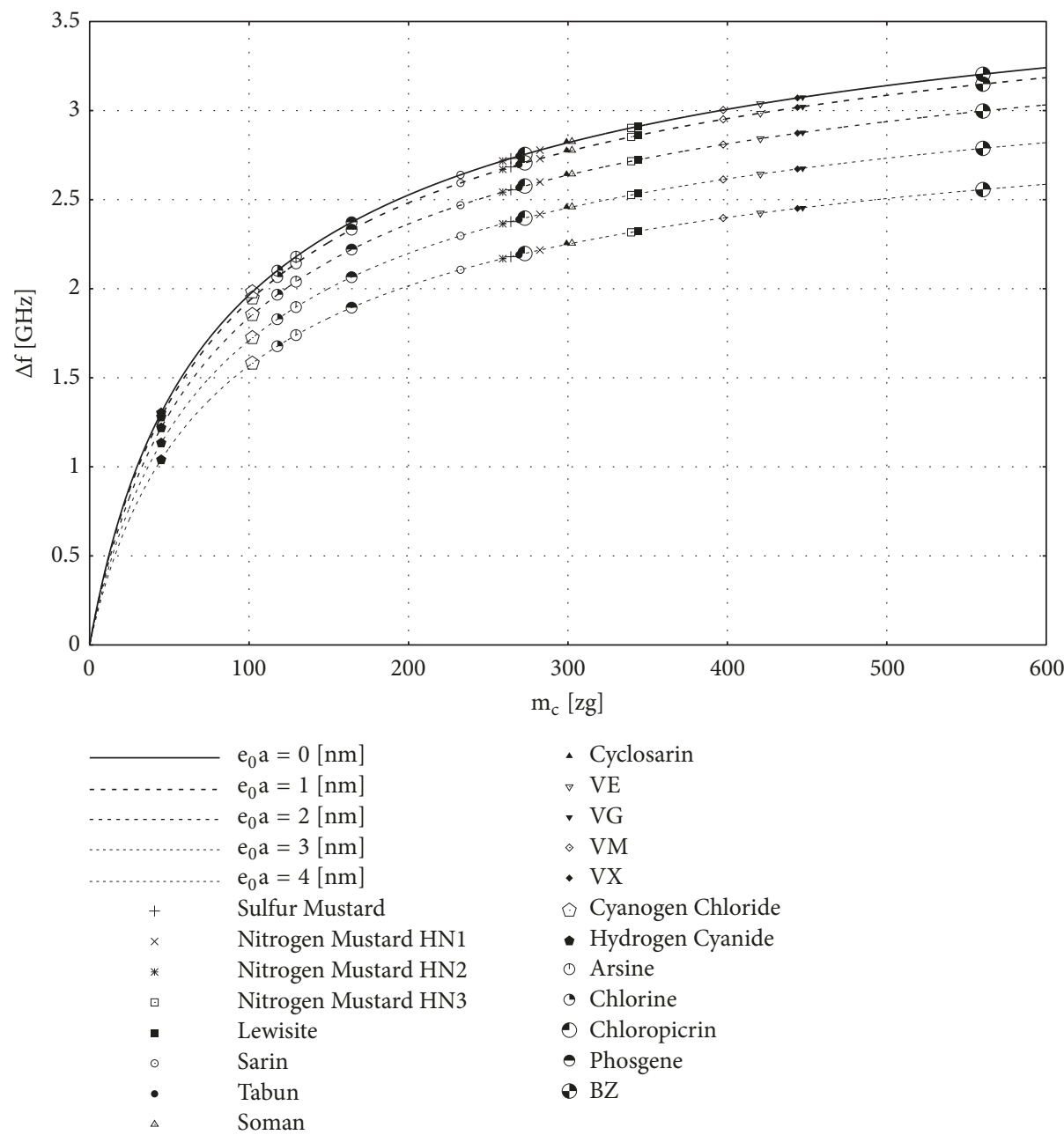

FIGURE 18: Frequency shift of simply supported SLGS versus mass, attached at SLGS center $(\xi=0.5, \eta=0.5, m=1, n=1)$. 


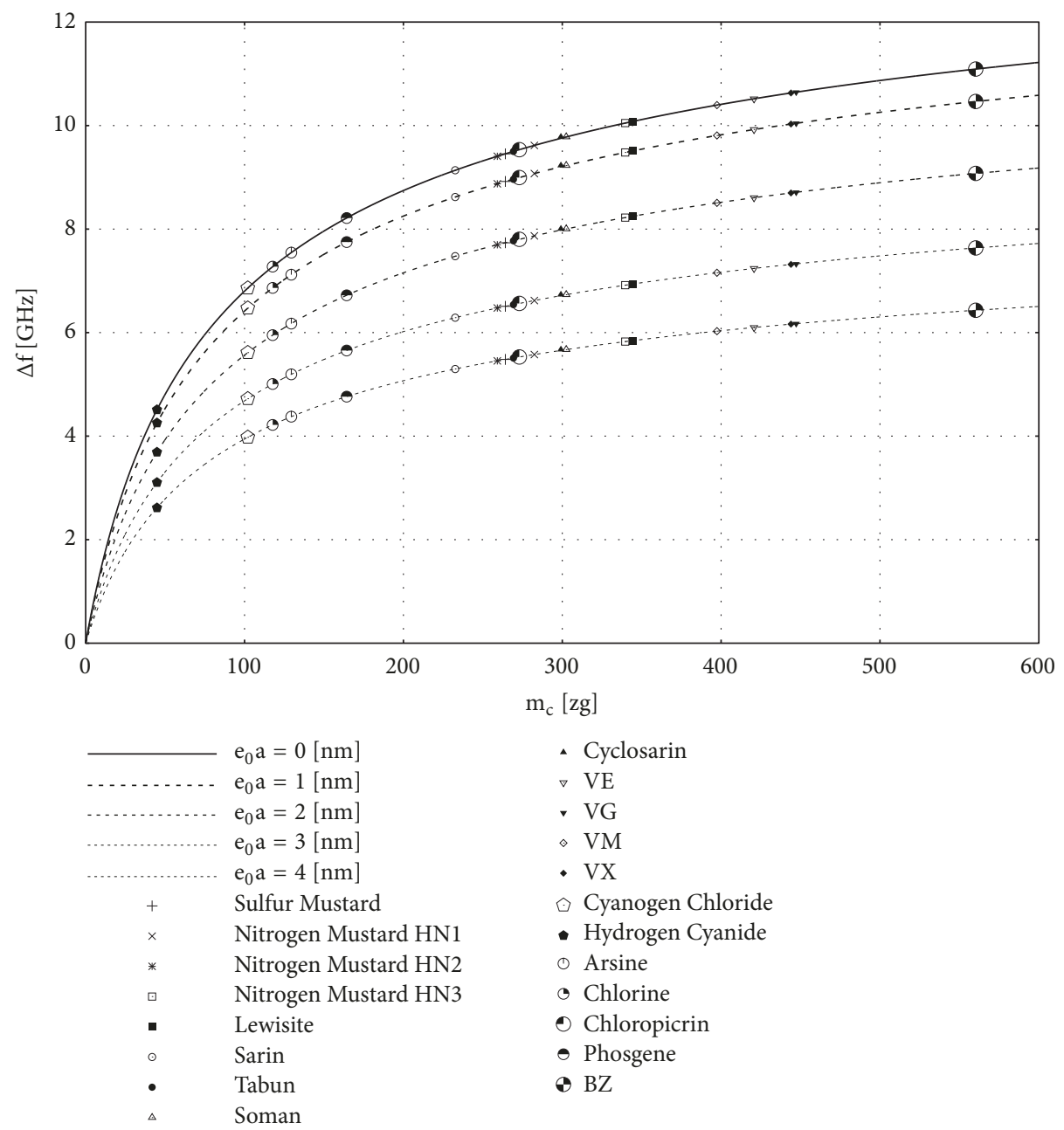

FIGURE 19: Frequency shift of simply supported SLGS versus mass, attached at SLGS center $(\xi=0.5, \eta=0.5, m=3, n=1)$.

and $L_{b}$ are in range from 40 to $160 \mathrm{~nm}$ the resonant frequency of DLGS is in range from 0 to $20 \mathrm{GHz}$. So as the dimensions of DLGS system increase, the frequencies of DLGS system decrease.

The next step is to show how the mass of attached nanoparticle and the nonlocal parameter together is influencing the resonant frequency of the simply supported DLGS system. As in the case of simply supported SLGS system the mass of attached nanoparticle is taken in range from 0 to $600 \mathrm{zg}$ since the mass CWA molecules from Table 2 are in that range. For this analysis the nonlocal parameter is taken in range from 0 to $100 \mathrm{~nm}$. The position of attached CWA nanoparticle is at the center of upper plate of DLGS $(\xi=$ $0.5, \eta=0.5)$. Nondimensional values of shape function of $m$ and $n$ are set to 1 and 2, respectively. The result of the analysis is shown in Figure 8.

From Figure 8 it is easy to conclude that mass of attached nanoparticle and nonlocal parameter lowers the resonant frequency of simply supported DLGS system. As in the case of simply supported SLGS the nonlocal parameter has greater influence on resonant frequency than the mass of attached nanoparticle.
3.3. Absolute and Relative Frequency Shift of Single-Layer and Double-Layer Graphene Sheets. In order to detect the attached nanoparticle by the absolute frequency shift method first the resonant frequencies of the SLGS or DLGS system without attached nanoparticle must be determined and then resonant frequencies with attached nanoparticle must be determined, respectively. Resonant frequencies of the free system and system with attached nanoparticle have to be determined since the frequency shift is defined as the difference between the resonant frequency of a SLGS (or DLGS) with and without attached nanoparticles. Thus, it can be written in the following form:

$$
\Delta f=f-f_{0},
$$

where $f_{0}$ is the resonant frequency of SLGS or DLGS without attached nanoparticle and $f$ the resonant frequency of SLGS or DLGS with attached nanoparticle. According to [32] relative frequency shift can be written in the following form:

$$
f_{\text {relative }}=\frac{\Delta f}{f_{0}}=\frac{f}{f_{0}}-1 .
$$




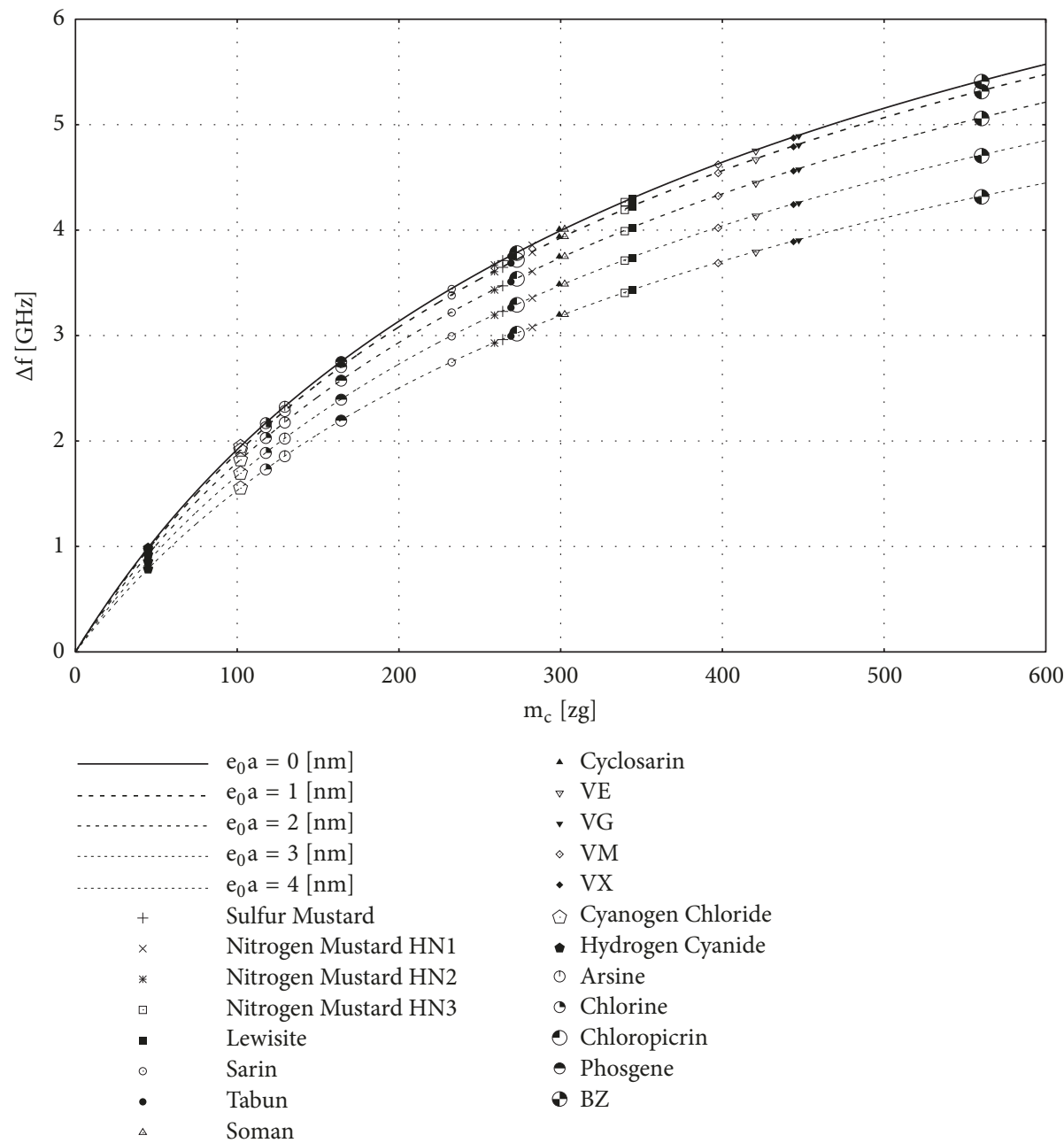

FIGURE 20: Frequency shift of the simply supported DLGS versus mass of attached mass at the center of upper plate $(\xi=0.5, \eta=0.5, m=$ $1, n=1)$.

From (38) it can be concluded that the nonlocal parameter has no influence on the relative frequency shift. In particular, since the term involving the nonlocal parameter in denominator $1+\left(e_{0} a\right)^{2} \pi^{2}\left(m^{2} / L_{a}^{2}+n^{2} / L_{b}^{2}\right)$ (17) can be canceled in division of frequencies with and without attached mass $f / f_{0}$, the relative frequency shift has to be independent of the nonlocal parameter. The same conclusion can be made for DLGS case, eq. (36). The importance of this observation is raised once more later in this section by means graphical representation.

3.3.1. Frequency Shift for Simply Supported SLGS System. As described above, frequency shifts for SLGS for different mode shapes are calculated and the results are provided in Figure 9.

From Figure 9 it can be seen that the mass of the attached CWA is not influencing all mode shapes. Attached CWA mass affects only first mode shape $(m=1, n=1)$, fifth mode shape $(m=3, n=1)$, and seventh mode shape $(m$ $=3, n=3$ ). In these mode shapes as the mass of attached nanoparticle increases the frequency shift increases too. The lack of influence of the frequency shift on other analyzed mode shapes can be explained by the position of attached mass. Since the attached nanoparticle is at the center of simply supported SLGS the molecule is located at the nodal line. The nodal line is the line which remains stationary during the vibration cycle. Since the attached nanoparticle is located on this nodal line there is no change in the resonant frequency or in the frequency shift. Figure 10 shows the difference between the mode shape without nodal line and mode shape with one nodal line.

In Figure 10 two mode shapes are shown. The first mode shape $(m=1, n=1)$ does not have nodal lines so the attached CWA molecule is influencing the resonant frequency. In second mode shape $(m=2, n=1)$ the attached CWA molecule is located on the nodal line which means that this molecule is not influencing the resonant frequency since the nodal line is not moving during the vibration cycle.

Since the mode shapes that reacted specific position of attached CWA molecule are found, the next step is to see how the nonlocal parameter is influencing the frequency shift of simply supported SLGS. The results of this analysis are shown in Figures 11-12. 


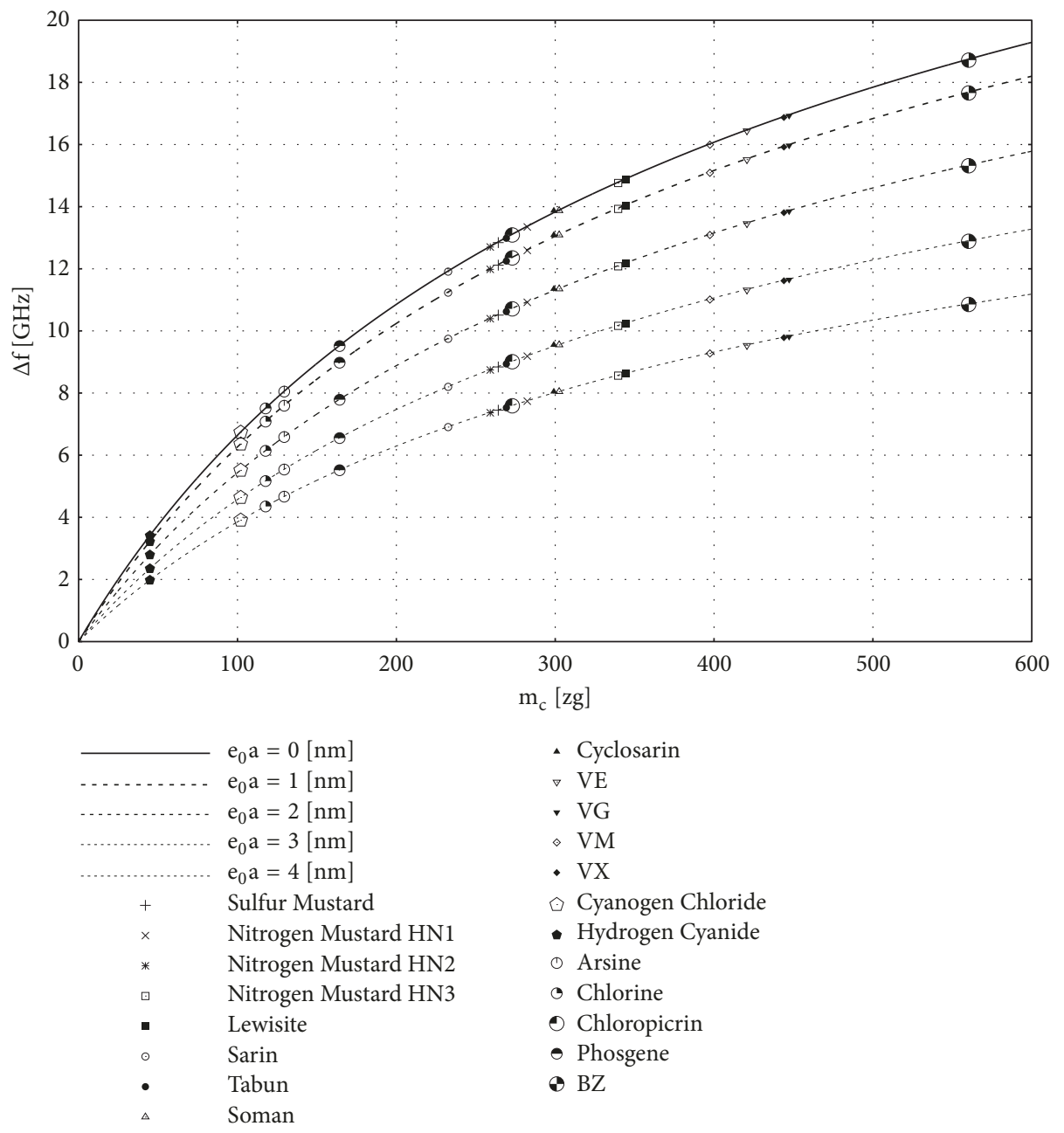

Figure 21: Frequency shift of the simply supported DLGS versus mass of attached mass at the center of upper plate $(\xi=0.5, \eta=0.5, m=$ $3, n=1)$.

As seen from Figures 11-12 the nonlocal parameter lowers the absolute frequency shift. When mass of nanoparticle is low the difference between resonant frequencies of SLGS without nanoparticle and SLGS with the attached nanoparticle is negligible. If a mass of attached nanoparticle becomes larger the difference in resonant frequencies between SLGS with and without attached nanoparticle becomes larger.

3.3.2. Influence of Attached CWA Molecule Location on the Frequency Shift for SLGS System. So far, the location of attached nanoparticle or CWA molecule was at a center of the simply supported SLGS system. The results of performed simulations showed that SLGS could detect a CWA molecule based on frequency shift method only for specific mode shapes. In this analysis the influence of attached CWA molecule location on frequency shift will be investigated for simply supported SLGS. This analysis was performed for mode shape with nondimensional mode shape parameters $m=2$ and $n=1$. This mode shape is a mode shape with one nodal line and the goal is to show that other mode shapes are also good for detection of CWA molecules but only when the CWA molecule is not attached on the nodal line. For this analysis two different CWA molecules are chosen, that is, hydrogen cyanide ( $\mathrm{CHN}$ ) and 3-quinuclidinyl benzilate (BZ). The mass of these two molecules represent the boundaries in the mass range of CWA molecules. Looking at Table 2 the CHN has lowest mass value of one molecule while BZ has the highest mass value. The results of the analysis are shown in Figures 13 and 14.

From Figures 13 and 14 it can be concluded that nondimensional location parameters also have the influence on the frequency shift and resonant frequency of simply supported SLGS system. As the values of nondimensional location parameters increase the value of frequency shift also increases. The specific mode shape used in this analysis showed that it can be used for detection of CWA molecules but not when these molecules are attached to nodal lines. As seen from Figures 13 and 14 the highest frequency shift is at the center of SLGS $(\xi=0.5, \eta=0.5)$.

3.3.3. Frequency Shift of Simply Supported DLGS System. The next step is to determine if a DLGS system could be possible 


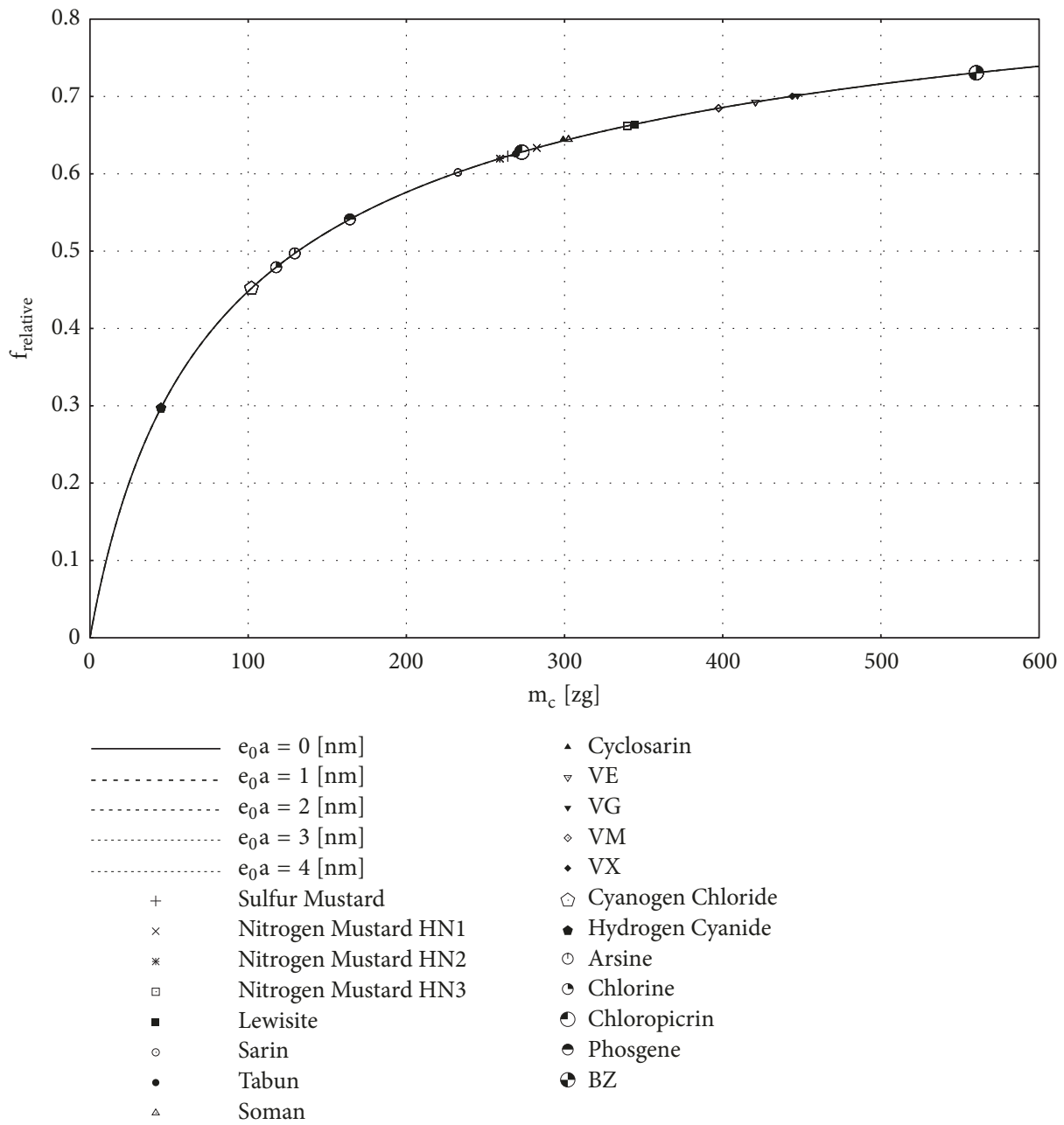

FigURE 22: Relative frequency shift of simply supported SLGS versus mass, attached at SLGS center $(\xi=0.5, \eta=0.5, m=1, n=1)$.

candidate for nanoparticle detection or detection of CWA molecules based on the absolute and relative frequency shift calculation. Like in case of simply supported SLGS, the mode shapes useful for detection of attached CWA molecules have to be identified. Results of the analysis are shown in Figure 15.

As seen in Figure 15 the frequency shift can be obtained for specific mode shapes of simply supported DLGS system and these are first mode shape $(m=1, n=1)$, fifth mode shape ( $m=3, n=1)$, and seventh mode shape ( $m=3, n$ $=3$ ). As in the case of simply supported SLGS the reason why some mode shapes could not detect the attached CWA molecule is because the attached CWA molecule is located on the nodal line. If the location of CWA molecule was changed then other mode shapes could be used for its detection. Now, mode shapes of the simply supported DLGS system that could detect the CWA molecules are found. The influence of the nonlocal parameter on the frequency shift is shown in Figures 16-17.

As in the case of simply supported SLGS system, the nonlocal parameter lowers the frequency shift for analyzed mode shapes of simply supported DLGS system. From previous figures it can also be concluded that for each of the following mode shapes the nonlocal parameter has greater influence on values of the frequency shift. For example, in first mode shape the influence of nonlocal parameter on the frequency shift is very small since the distance between the frequency shift curves is very small, Figure 16. Looking at Figure 17 it can be concluded that the distance between each of the frequency shift curves is larger when compared to the first mode shape so the influence of the nonlocal parameter is not the same for different mode shapes.

3.4. Detection of Chemical Warfare Agents with Single-Layer and Double-Layer Graphene Sheets Based on the Frequency Shift Method. In this analysis the simply supported SLGS is modelled based on the mechanical and geometrical properties in Table 1. Mass of the attached nanoparticle is now replaced with the CWA molecules which are presented in Table 2. The results of the analysis are shown in Figures 1819.

From Figures 18-19 it can be concluded that simply supported SLGS system is capable of detecting all of the CWA molecules given in Table 2 by the application of the frequency shift method. 


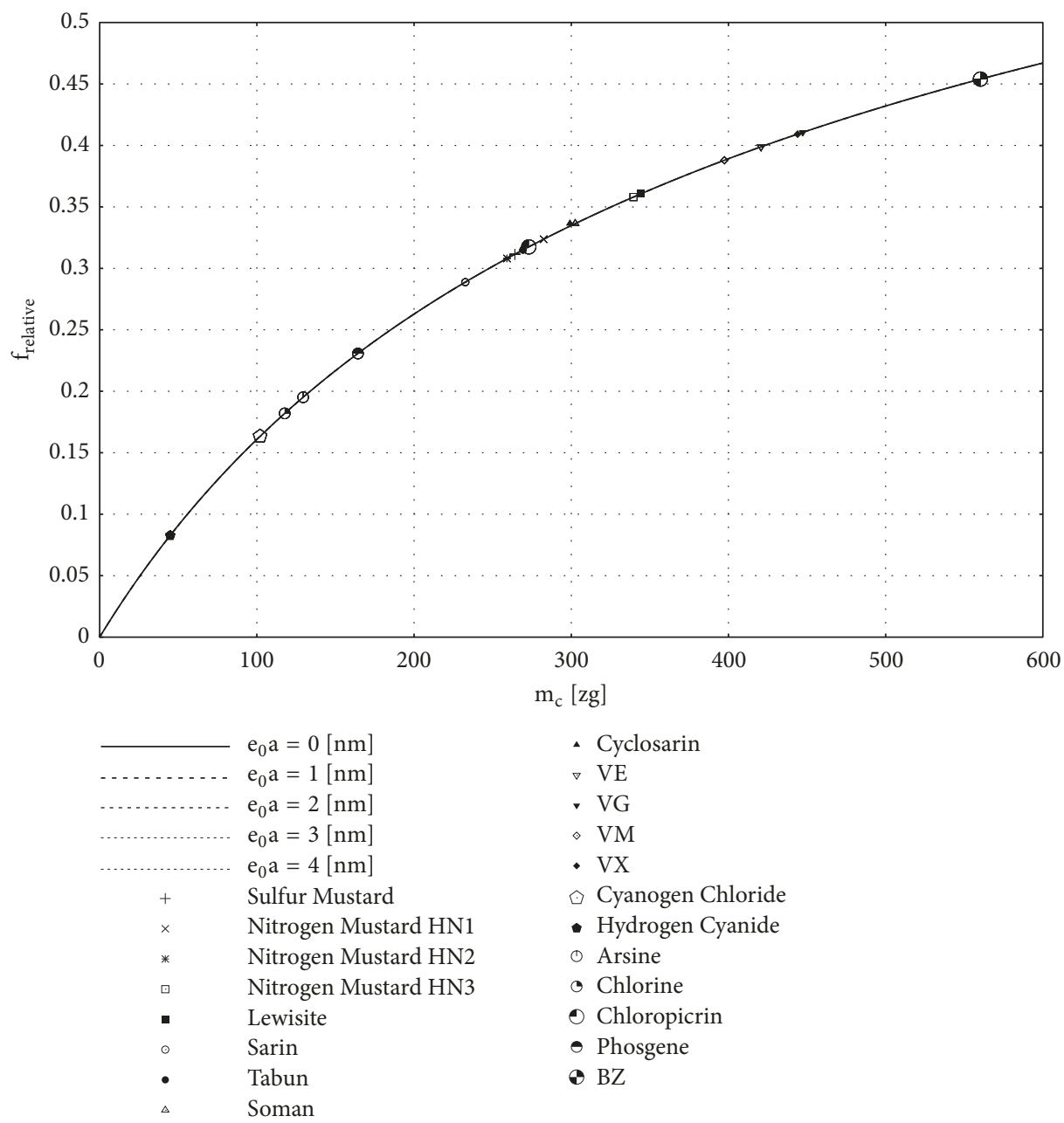

FIGURE 23: Relative frequency shift of simply supported SLGS versus mass, attached at DLGS center $(\xi=0.5, \eta=0.5, m=1, n=1)$.

The simply supported DLGS system is also modeled based on the mechanical and geometrical data in Table 1. The CWA molecule is attached at the upper plate of DLGS system at its center. The analysis is conducted for first $(m=1, n=1)$ and fifth $(m=3, n=1)$ mode shape since they are sensitive to the frequency shift. The results of the performed analysis are shown in Figures 20-21.

Based on results shown in Figures 20-21 the specific mode shapes of simply supported DLGS system can detect the CWA molecules given in Table 2 based on the frequency shift method.

3.5. Detection of Chemical Warfare Agents with SingleLayer and Double-Layer Graphene Sheets Based on Relative Frequency Shift Method. In this analysis the possibility of the SLGS and DLGS system in detection of CWA will be investigated using relative frequency shift method. As in the case of frequency shift (absolute) analysis the geometrical and material data of the SLGS and DLGS system are taken from Table 2. The position of CWA molecule is at center of SLGS and center of upper plate of DLGS. The investigation will be conducted only for the first mode shape $(m=1, n=1)$ since the values of relative frequency shift are the same for other two modes and there is no influence of the nonlocal parameter on relative frequency shift as described before. The results of analysis are shown in Figures 22-23.

In Figures 22 and 23 the variation of relative frequency is shown as a function of attached nanoparticle at the center of the SLGS and upper DLGS plate. These figures also show that all CWA molecules line on the relative frequency shift curve which means they could be detected. The results obtained for the absolute and relative frequency shift showed that SLGS and DLGS plate can detect CWA molecules.

\section{Conclusions}

In this paper, the resonant frequencies of simply supported SLGS and DLGS were analyzed by the nonlocal plate theory. The nonlocal plate theory, being an extension of the classical plate theory (Kirchhoff) can be used to tackle problems at nanoscale. The results showed the following.

(i) The nonlocal parameter has a huge influence on resonant frequencies of simply supported SLGS and 
DLGS. As demonstrated, maximal natural frequencies are obtained for the local solutions. When the nonlocal parameter gets larger than zero, the natural frequency is lowered. The nonlocal parameter generally tends to lower natural or resonant frequencies of SLGS and DLGS for every mode shape.

(ii) For small dimensions of SLGS or DLGS the natural or resonant frequencies in $\mathrm{MHz}$ range (obtained in experimental investigations $[19,50,51])$ could be obtained by the nonlocal theory in the absence of dissipation mechanisms, only if the nonlocal parameter is greater than $20 \mathrm{~nm}$.

(iii) For the large values of the nonlocal parameter and the attached nanoparticle mass the resonant frequencies of the SLGS and DLGS systems are in the MHz range as in $[19,50,51]$,

(iv) The influence of geometry of simply supported SLGS or DLGS system showed that as dimensions of plates increase the frequency drops to a certain value and after that it remains constant.

(v) The investigation also showed that the mass of attached nanoparticle has huge influence on resonant frequencies of simply supported SLGS and DLGS. As the mass of the attached nanoparticle increases the resonant frequencies become lower.

The central investigation in this paper was calculation of the frequency shift. In order to build fully functional NEMS where GS is a building block for detection of CWA molecules, the resonant frequency of SLGS or DLGS with and without CWA molecules needs to be determined. The results showed the following.

(i) Based on the difference of resonant frequencies free SLGS or DLGS and resonant frequency of SLGS or DLGS with attached CWA, it can be determined which CWA molecule is attached to the system.

(ii) The position of attached CWA on SLGS and DLGS system is also important. In this paper the position of CWA molecule was at the center of simply supported SLGS and DLGS system and the analysis showed that only certain mode shapes could detect CWA molecules. The main reason for this anomaly stems from the stationarity of nodal lines during the vibration cycle, so the mass of CWA molecule has no influence on the resonant frequency or the frequency shift of system. So, in order to use SLGS or DLGS system as a building block for NEMS mass resonators analysis of mode shapes which are capable of detection of CWA molecules must be conducted and the frequency shift method must be applied. This will provide information about the specific CWA molecule which is attached to GS. In this paper the position of CWA molecules on frequency shift method was also investigated and results showed that other nonactive mode shapes could be utilized. It is shown that both SLGS and DLGS systems can be highly effective in such detection. (iii) The nonlocal parameter does not affect relative frequency shift regardless of mode shape. The result showed that the mode shapes that showed change in absolute frequency shift versus added mass have equal relative frequency changes.

\section{Data Availability}

The data used to support the findings of this study are available from the corresponding author upon request.

\section{Conflicts of Interest}

The authors declare that they have no conflicts of interest.

\section{Acknowledgments}

This work has been supported in part by the University of Rijeka under the project numbers uniri-tehnic-18-37 and uniri-tehnic-18-275-1447 and partly supported by the CEEPUS network CIII-HR-0108 and European Regional Development Fund under the grant KK.01.1.1.01.0009 (DATACROSS).

\section{References}

[1] T. Natsuki, "Theoretical analysis of vibration frequency of graphene sheets used as nanomechanical mass sensor," Electronics, vol. 4, no. 4, pp. 723-738, 2015.

[2] A. Montagner, S. Bosi, E. Tenori et al., "Ecotoxicological effects of graphene-based materials," 2D Materials, vol. 4, no. 1, Article ID 012001, 2016.

[3] Y. Volkov, J. McIntyre, and A. Prina-Mello, "Graphene toxicity as a double-edged sword of risks and exploitable opportunities: a critical analysis of the most recent trends and developments," 2D Materials, vol. 4, no. 2, Article ID 022001, 2017.

[4] T. Natsuki, J.-X. Shi, and Q.-Q. Ni, "Vibration analysis of nanomechanical mass sensor using double-layered graphene sheets resonators," Journal of Applied Physics, vol. 114, no. 9, Article ID 094307, 2013.

[5] Q.-T. Vien, M. O. Agyeman, T. A. Le, and T. Mak, "On the nanocommunications at $\mathrm{THz}$ band in graphene-enabled wireless network-on-chip," Mathematical Problems in Engineering, vol. 2017, Article ID 9768604, 13 pages, 2017.

[6] A. K. Naik, M. S. Hanay, W. K. Hiebert, X. L. Feng, and M. L. Roukes, "Towards single-molecule nanomechanical mass spectrometry," Nature Nanotechnology, vol. 4, no. 7, pp. 445450, 2009.

[7] K. Jensen, K. Kim, and A. Zettl, "An atomic-resolution nanomechanical mass sensor," Nature Nanotechnology, vol. 3, no. 9, pp. 533-537, 2008.

[8] T. P. Burg, M. Godin, S. M. Knudsen et al., "Weighing of biomolecules, single cells and single nanoparticles in fluid," Nature, vol. 446, no. 7139, pp. 1066-1069, 2007.

[9] H. Arjmandi-Tash, A. Allain, Z. Han, and V. Bouchiat, "Large scale integration of CVD-graphene based NEMS with narrow distribution of resonance parameters," 2D Materials, vol. 4, no. 2, Article ID 025023, 2017. 
[10] D. Miller and B. Alemán, "Shape tailoring to enhance and tune the properties of graphene nanomechanical resonators," 2D Materials, vol. 4, no. 2, Article ID 025101, 2017.

[11] S. S. Varghese, S. Lonkar, K. K. Singh, S. Swaminathan, and A. Abdala, "Recent advances in graphene based gas sensors," Sensors and Actuators B: Chemical, vol. 218, pp. 160-183, 2015.

[12] M. Donarelli, S. Prezioso, F. Perrozzi et al., "Graphene oxide for gas detection under standard humidity conditions," $2 D$ Materials, vol. 2, no. 3, Article ID 035018, 2015.

[13] C. Zhu, D. Du, and Y. Lin, "Graphene and graphene-like 2D materials for optical biosensing and bioimaging: a review," $2 D$ Materials, vol. 2, no. 3, Article ID 032004, 2015.

[14] A. Y. Zhu and E. Cubukcu, "Graphene nanophotonic sensors," 2D Materials, vol. 2, no. 3, Article ID 032005, 2015.

[15] Y. T. Yang, C. Callegari, X. L. Feng, K. L. Ekinci, and M. L. Roukes, "Zeptogram-scale nanomechanical mass sensing," Nano Letters, vol. 6, no. 4, pp. 583-586, 2006.

[16] H. Boo, R.-A. Jeong, S. Park et al., "Electrochemical nanoneedle biosensor based on multiwall carbon nanotube," Analytical Chemistry, vol. 78, no. 2, pp. 617-620, 2006.

[17] Z. Chen, Z. Wang, X. Li et al., "Flexible piezoelectricinduced pressure sensors for static measurements based on nanowires/graphene heterostructures," ACS Nano, vol. 11, no. 5, pp. 4507-4513, 2017.

[18] G. Lu, L. E. Ocola, and J. Chen, "Reduced graphene oxide for room-temperature gas sensors," Nanotechnology, vol. 20, no. 44, pp. 445502-4455011, 2009.

[19] C. Yang, A. Mahmood, B. Kim et al., "Enhancing gas sensing properties of graphene by using a nanoporous substrate," $2 D$ Materials, vol. 3, no. 1, Article ID 011007, 2016.

[20] S. Rumyantsev, G. Liu, M. S. Shur, R. A. Potyrailo, and A. A. Balandin, "Selective gas sensing with a single pristine graphene transistor," Nano Letters, vol. 12, no. 5, pp. 2294-2298, 2012.

[21] B. Huang, Z. Li, Z. R. Liu et al., "Adsorption of gas molecules on graphene nanoribbons and its implication for nanoscale molecule sensor," The Journal of Physical Chemistry C, vol. 112, no. 35, pp. 13442-13446, 2008.

[22] S. Some, Y. Xu, Y. Kim et al., "Highly sensitive and selective gas sensor using hydrophilic and hydrophobic graphenes," Scientific Reports, vol. 3, p. 1868, 2013.

[23] Organization for the Prohibition of Chemical Weapons (OPCW), "Convention on the prohibition of the development, production, stockpiling and use of chemical weapons and on their destruction," Technical Secretariat of the OPCW, 2005.

[24] S. Chauhan, S. Chauhan, R. D'Cruz et al., "Chemical warfare agents," Environmental Toxicology and Pharmacology, vol. 26, no. 2, pp. 113-122, 2008.

[25] R. Barretta, M. Brčić, M. Čanađija, R. Luciano, and F. Marotti de Sciarra, "Application of gradient elasticity to armchair carbon nanotubes: size effects and constitutive parameters assessment," European Journal of Mechanics - A/Solids, vol. 65, pp. 1-13, 2017.

[26] R. Ansari, S. Sahmani, and B. Arash, "Nonlocal plate model for free vibrations of single-layered graphene sheets," Physics Letters A, vol. 375, no. 1, pp. 53-62, 2010.

[27] R. Barretta, R. Luciano, and F. Marotti de Sciarra, "A fully gradient model for euler-bernoulli nanobeams," Mathematical Problems in Engineering, vol. 2015, Article ID 495095, 8 pages, 2015.

[28] M. Čanadija, R. Barretta, and F. M. de Sciarra, "On functionally graded Timoshenko nonisothermal nanobeams," Composite Structures, vol. 135, pp. 286-296, 2016.
[29] F. Marotti de Sciarra, M. Canadija, and R. Barretta, "A gradient model for torsion of nanobeams," Comptes Rendus Mécanique, vol. 343, no. 4, pp. 289-300, 2015.

[30] M. Čanađija, R. Barretta, and F. Marotti de Sciarra, "A gradient elasticity model of Bernoulli-Euler nanobeams in nonisothermal environments," European Journal of Mechanics A/Solids, vol. 55, pp. 243-255, 2016.

[31] L. Shen, H.-S. Shen, and C.-L. Zhang, "Nonlocal plate model for nonlinear vibration of single layer graphene sheets in thermal environments," Computational Materials Science, vol. 48, no. 3 , pp. 680-685, 2010.

[32] R. Barretta, M. Čanadija, L. Feo, R. Luciano, F. Marotti de Sciarra, and R. Penna, "Exact solutions of inflected functionally graded nano-beams in integral elasticity," Composites Part B: Engineering, vol. 142, pp. 273-286, 2018.

[33] R. Barretta, M. Čanađija, R. Luciano, and F. Marotti de Sciarra, "Stress-driven modeling of nonlocal thermoelastic behavior of nanobeams," International Journal of Engineering Science, vol. 126, pp. 53-67, 2018.

[34] E. Jomehzadeh and A. R. Saidi, "A study on large amplitude vibration of multilayered graphene sheets," Computational Materials Science, vol. 50, no. 3, pp. 1043-1051, 2011.

[35] D. Karlicic, T. Murumu, S. Adhikari, and M. McCarthy, Nonlocal Structural Mechanics, John Wiley \& Sons, Inc, 2016.

[36] A. Bouadi, A. A. Bousahla, M. S. A. Houari, H. Heireche, and A. Tounsi, "A new nonlocal HSDT for analysis of stability of single layer graphene sheet," Advances in Nano Research, vol. 6, no. 2, pp. 147-162, 2018.

[37] Y. Mokhtar, H. Heireche, A. A. Bousahla, M. S. A. Houari, A. Tounsi, and S. R. Mahmoud, "A novel shear deformation theory for buckling analysis of single layer graphene sheet based on nonlocal elasticity theory," Smart Structures and Systems, vol. 21, no. 4, pp. 397-405, 2018.

[38] S. Gopalakrishnan and S. Narendar, Wave Propagation in Nanostructures: Nonlocal Continuum Mechanics Formulations, Springer Science Business Media, 2013.

[39] ChemSpider, 2018, http://www.chemspider.com/.

[40] S. Kitipornchai, X. Q. He, and K. M. Liew, "Continuum model for the vibration of multilayered graphene sheets," Physical Review B: Condensed Matter and Materials Physics, vol. 72, no. 7, Article ID 075443, 2005.

[41] X. Q. He, S. Kitipornchai, and K. M. Liew, "Buckling analysis of multi-walled carbon nanotubes: a continuum model accounting for van der Waals interaction," Journal of the Mechanics and Physics of Solids, vol. 53, no. 2, pp. 303-326, 2005.

[42] X. Q. He, S. Kitipornchai, and K. M. Liew, "Resonance analysis of multi-layered graphene sheets used as nanoscale resonators," Nanotechnology, vol. 16, no. 10, pp. 2086-2091, 2005.

[43] F. Memarian, A. Fereidoon, and M. Darvish Ganji, "Graphene Young's modulus: Molecular mechanics and DFT treatments," Superlattices and Microstructures, vol. 85, pp. 348-356, 2015.

[44] J. Jiang, J. Wang, and B. Li, "Young's modulus of graphene: a molecular dynamics study," Physical Review B: Condensed Matter and Materials Physics, vol. 80, no. 11, Article ID 113405, 2009.

[45] N. Jing, Q. Xue, C. Ling et al., "Effect of defects on young's modulus of graphene sheets: a molecular dynamics simulation," RSC Advances, vol. 2, no. 24, pp. 9124-9129, 2012.

[46] M. M. Shokrieh and R. Rafiee, "Prediction of Young's modulus of graphene sheets and carbon nanotubes using nanoscale continuum mechanics approach," Materials and Corrosion, vol. 31, no. 2, pp. 790-795, 2010. 
[47] R. Rafiee and A. Eskandariyun, "Comparative study on predicting Young's modulus of graphene sheets using nano-scale continuum mechanics approach," Physica E: Low-dimensional Systems and Nanostructures, vol. 90, pp. 42-48, 2017.

[48] P. Lengvarský and J. Bocko, "Prediction of Youngs modulus of graphene sheets by the finite element method," American Journal of Mechanical Engineering, vol. 3, pp. 225-229, 2015.

[49] J.-U. Lee, D. Yoon, and H. Cheong, "Estimation of young's modulus of graphene by Raman spectroscopy," Nano Letters, vol. 12, no. 9, pp. 4444-4448, 2012.

[50] C. Chen, S. Rosenblatt, K. I. Bolotin et al., "Performance of monolayer graphene nanomechanical resonators with electrical readout," Nature Nanotechnology, vol. 4, no. 12, pp. 861-867, 2009.

[51] C. Chen, S. Lee, V. V. Deshpande et al., "Graphene mechanical oscillators with tunable frequency," Nature Nanotechnology, vol. 8, no. 12, pp. 923-927, 2013. 


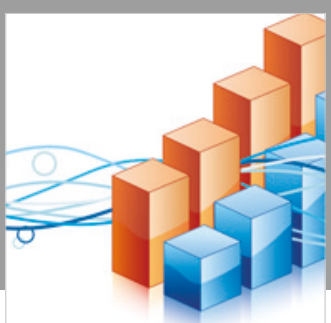

Advances in

Operations Research

\section{-n-m}
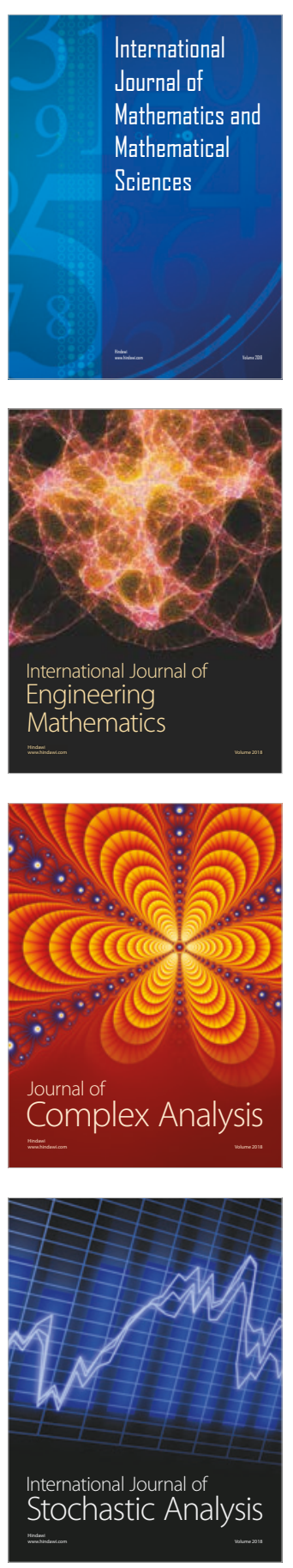
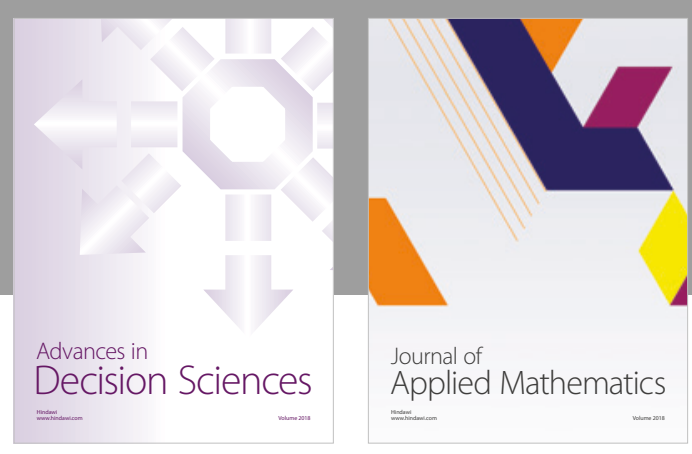

Journal of

Applied Mathematics
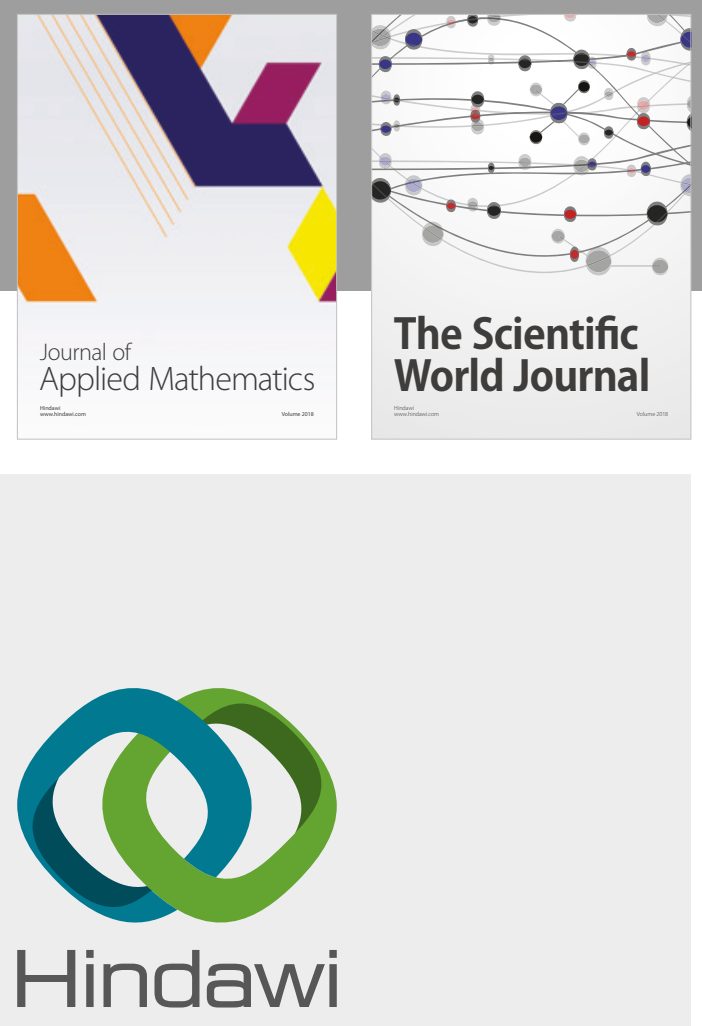

Submit your manuscripts at

www.hindawi.com

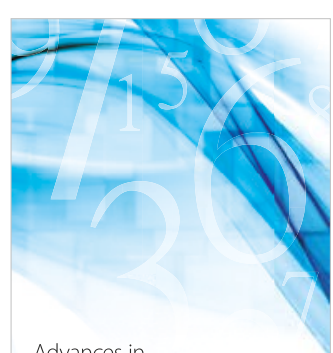

Advances in
Numerical Analysis
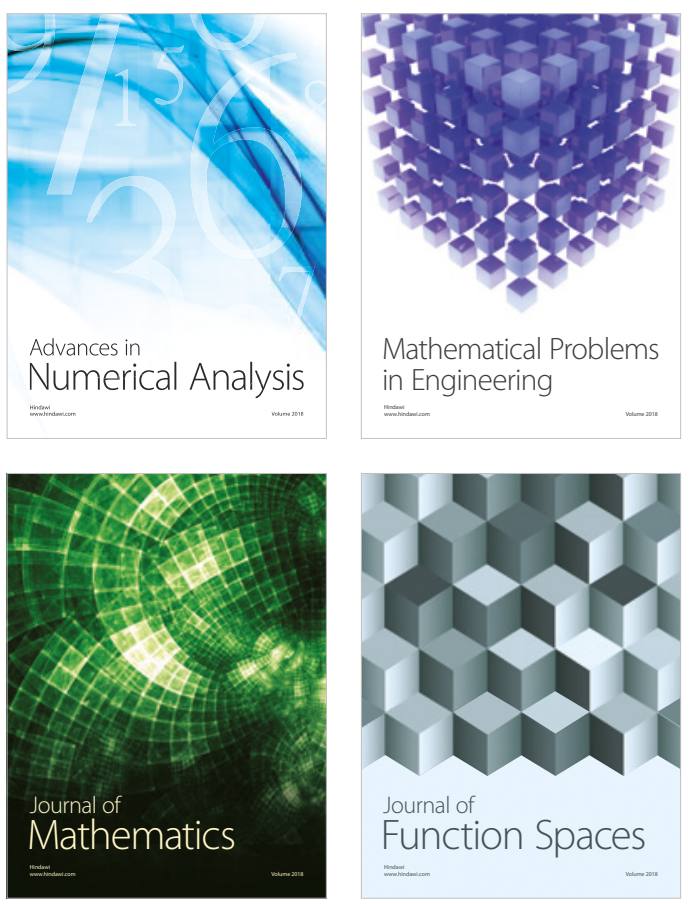

Mathematical Problems in Engineering

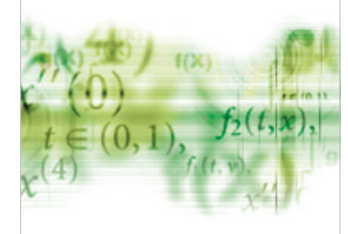

International Journal of

Differential Equations

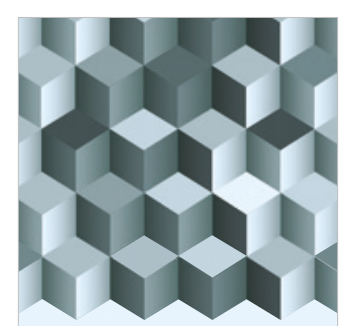

Journal of

Function Spaces

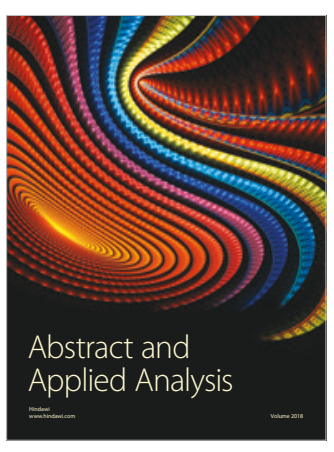

The Scientific

World Journal

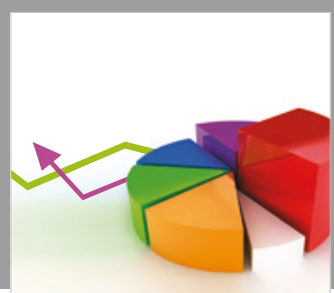

Journal of

Probability and Statistics
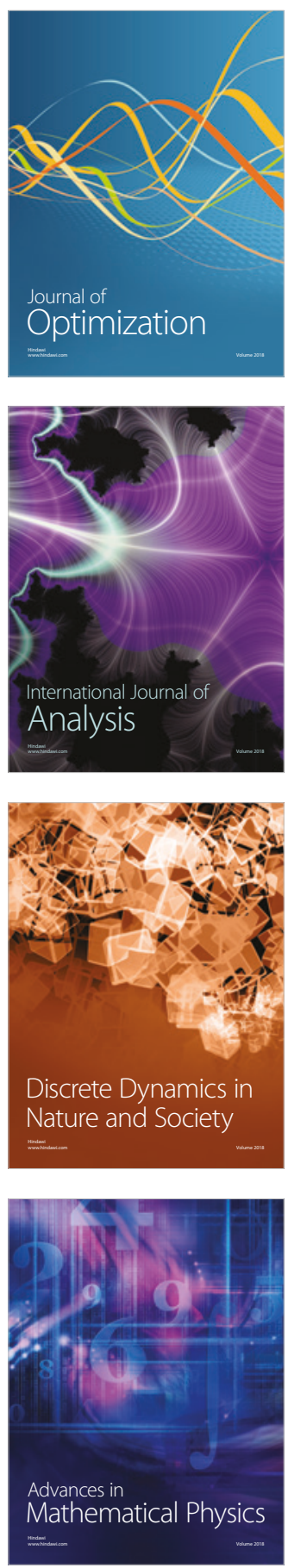